\title{
Prediction of diffuse horizontal irradiance using a new climate zone model
}

\author{
Edgar F.M. Abreu ${ }^{\mathrm{a}, *}$, Paulo Canhoto ${ }^{\mathrm{a}, \mathrm{b}}$, Maria João Costa ${ }^{\mathrm{a}, \mathrm{b}}$ \\ ${ }^{a}$ Instituto de Ciências da Terra, Universidade de Évora, Rua Romão Ramalho, 59, 7000-671, Évora, Portugal \\ ${ }^{\mathrm{b}}$ Departamento de Física, Escola de Ciências e Tecnologia, Universidade de Évora, Rua Romão Ramalho 59, 7000-671, Évora, Portugal
}

A R T I C L E I N F O

\section{Keywords:}

Diffuse horizontal irradiance

Global horizontal irradiance

Direct normal irradiance

Clearness index

Diffuse fraction

Separation method

\begin{abstract}
A B S T R A C T
Knowledge on the diffuse horizontal irradiance (DHI), and direct normal irradiance (DNI) is crucial for the estimation of the irradiance on tilted surfaces, which in turn is critical for photovoltaic (PV) applications and for designing and simulating concentrated solar power (CSP) plants. Since global horizontal irradiance (GHI) is the most commonly measured solar radiation variable, it is advantageous for establishing a suitable method that uses it to compute DHI and DNI. In this way, a new model for predicting the diffuse fraction $\left(K_{d}\right)$ based on the climate zone is proposed, using only the clearness index $\left(K_{t}\right)$ as the predictor and 1-min resolution GHI data. A review of the literature on models that use hourly and sub-hourly $K_{t}$ values to compute $K_{d}$ was also carried out, and an extensive performance assessment of both the proposed model and the models from the literature was conducted using ten statistical indicators and a global performance index (GPI). A set of model parameters was determined for each climate zone considered in this study (arid, high albedo, temperate and tropical) using 48 worldwide radiometric stations. It was found that the best overall performing model was the model proposed in this work.
\end{abstract}

\section{Introduction}

Global horizontal irradiance (GHI) is the most commonly measured solar radiation variable in the ground-based meteorological stations around the world, both in historical datasets and in geographical distribution. Therefore, it is the best dataset available to quantify solar energy resource and assess undergoing or future solar energy projects. On the other hand, information on both diffuse horizontal irradiance (DHI) and direct normal irradiance (DNI) is also crucial to properly design and optimise solar energy systems. In this way, it is advantageous to find a suitable and accurate method based on the GHI measurements to estimate both DHI and DNI, thus enabling the reconstitution of temporal series of these two components in locations where only GHI measurements are available, mainly due to budget limitations and higher requirements for maintenance and calibration procedures. In fact, whereas pyranometer installations are relatively cheap (USD 5$10 \mathrm{~K}$ with a data logger), full stations equipped with a sun tracker, pyranometers and a pyrheliometer are quite expensive (around USD $30 \mathrm{~K}$ ) [1]. DHI and DNI data are essential to accurately determine the global solar irradiance on tilted surfaces, for example in sizing and operation of photovoltaic (PV) systems [2]. The models for the diffuse fraction allow to estimate those components based on the GHI and then determine the irradiance on a tilted surface, by opposition to the one- step methods of converting GHI, as for example the isotropic sky model [3], the Klucher model [4], the Hay-Davies model [5] and the Reindl model [6]. Concentrated Solar Power (CSP) systems mainly use DNI in its energy capturing and conversion processes due to its directional nature and field of view (aperture angle) that depends on the concentration factor [7]. Therefore, the accurate computation of DHI is of vital importance to design, assess the performance and operate such systems [8].

The response of the scientific community for the need of obtaining DHI and DNI data at low cost was given by developing separation models in which the work of Liu and Jordan [9] was the pioneer. That work reported the relation between the clearness index (the ratio between GHI that reaches the surface of the earth and the extraterrestrial irradiance on a horizontal surface, $K_{t}$ ) and the diffuse fraction (the ratio of DHI to GHI, $K_{d}$ ) using measurements from 98 stations in Canada and United States. The good results obtained by Liu and Jordan lead to the development of several other separation models for different locations. Page [10] developed a model based on monthly mean values for latitudes between $40^{\circ} \mathrm{N}$ and $40^{\circ} \mathrm{S}$. Tuller [11] analysed daily and monthly data to establish models for four locations in Canada. Klein [12] used experimental measurements to assess and validate the model proposed by Liu and Jordan [9] and extended it to allow calculation of monthly average solar irradiation on surfaces with multiple orientations.

\footnotetext{
* Corresponding author.

E-mail address: eabreu@uevora.pt (E.F.M. Abreu).
} 
Although several other daily and monthly basis models were presented and are available in the literature, they are not the focus of this work. The work of Khorasanizadeh and Mohammadi [13] reports a comprehensive review of such models. Separation models for high-frequency GHI data are needed until high-resolution DNI measurements are available in a global scale, since required temporal resolution of nowadays reported solar radiation data increased, due to the requirements of high-frequency measurements in the simulation of CSP projects [14]. Therefore, because sub-hourly models are relatively rare in the literature, this work focuses on the available hourly and sub-hourly separation models whose solo predictor is the $K_{t}$ and on their ability in representing high-frequency data in a global scale and for different climates. The main reason for using only $K_{t}$ as the predictor is due to the greater availability of GHI data worldwide, thus allowing a straightforward evaluation of the model for the higher number of locations as possible. Regarding this type of models that use only $K_{t}$ as the predictor, Orgill and Hollands [15] presented a separation model using hourly measurements covering the period from September 1967 to August 1971 for Toronto Airport, Canada. This was the first model found in the literature that met the features mentioned above. In Section 2 are presented all the other models reviewed in this work.

The assessment of new separation models is usually carried out through the comparison of that new model against ground measurements and other models [1] using statistical indicators. Beside some researchers have already presented performance analysis using only models available in the literature $[14,16,17]$, the majority of the validation studies were reported when new models were derived, as is the case of this work. The first hourly models presented [15,18] were compared against the Liu and Jordan monthly model [9]. As time went by, more hourly models became available for test, and therefore models such as the Orgill and Hollands [15] and the Erbs et al. [19] were used in numerous validation studies (e.g. Refs. $[6,20,21]$ ). Liu and Jordan's model is still occasionally used with the purpose of presenting a historical comparison of the separation models evolution [22]. Regarding the validation using ground-based measurements, the most used statistical indicators to assess the performance of separation models are the mean bias error (MBE), the root mean square error (RMSE) and the correlation coefficient (R).

One-minute data resolution models are very scarce in the literature. One of the few examples is the work of Engerer [1], which presents a diffuse fraction model based on 1-min clearness index data together with other predictors for southeastern Australia. Gueymard and RuizArias [14] reported the incapability of hourly models to account for cloud enhancement effects, aiming at the need for reliability in hourly models until more specific minutely models appear in the literature. Therefore, the purpose of this study is to develop a new diffuse fraction model based on 1-min measurements from stations around the globe. Since the model presented by Engerer [1] requires more than one input parameter, the performance assessment of the model developed in this work will be conducted against hourly and sub-hourly models whose only predictor is $K_{t}$. To that end, ten statistical indicators were used, namely the mean bias error (MBE), mean absolute error (MAE), root mean square error (RMSE), mean percentage error (MPE), uncertainty at 95\% (U95), relative root mean square error (RRMSE), maximum absolute error (erMAX), correlation coefficient (R) and mean absolute relative error (MARE). These statistical indicators were also combined into a global performance index (GPI). The GPI was used in previous studies in this field by Jamil and Akhtar [23]. Other option to combine different statistical indicators is the combined performance index (CPI), as described by Gueymard [24]. A Taylor diagram and a skill score [25] were also used to provide an additional statistical analysis. In this view, a comprehensive performance assessment of the proposed model as well as of other models in the literature is presented aiming at the identification of the best performing model for the estimation of DHI in a minute resolution all over the world. The organization of this paper is as follows: Section 2 presents a review of the hourly and sub-hourly models for estimating the diffuse fraction, Section 3 presents the data used in this study and the model development, Section 4 presents the results and discussion, and, finally, conclusions are drawn in Section 5.

\section{Review of the available models}

The models available in the literature were developed using several functional forms, number of predictors and for different time resolutions. The first form to obtain the diffuse fraction was a second degree polynomial as a function of the clearness index, as first proposed by Liu and Jordan [9] in 1960. Later, other models were presented using higher polynomial degrees as well as other functions such as the logistic [26] and the double exponential [27] forms. Several models included other predictors than $K_{t}$, such as sunshine duration, zenith angle, air mass, etc. Regarding time resolution, the available models were proposed to estimate the monthly, daily, hourly and sub-hourly diffuse fraction. In this work, a review of the models that use only $K_{t}$ as the predictor with hourly or sub-hourly time resolutions is presented. The authors were able to find 121 different models that met the requirements specified above, although more models may be available in other publications or internal reports and communications that are not readily accessible. In many cases, authors present the same model but for different locations. These models are treated here as unique models when assessing their performance in Section 4. Table 1 presents the models studied in this work. The various locations from which authors used data to develop their models are classified according to the climate region as follows [14]: temperate (TM), arid (AR), tropical (TR) and high albedo (HA).

Based on the information of Table 1, it is notorious that the majority of the models were derived using a polynomial form, followed by the double exponential and logistic functions. This information is also useful to see the distribution of the data used to derive the models according to the climate zone and to identify the length of the datasets, as this is a crucial factor on the determination of the coefficients of the models. Fig. 1 presents the distribution of models as a function of the number of years of the datasets and climate zone. Only the models that presented an explicit dataset length in their respective publications were used to produce Fig. 1. Models based on data from several locations, different climatic zones or models derived from distinct dataset lengths were not considered, hence the 100 models examined in Fig. 1. The higher number of models was developed for the temperate (TM) zone, followed by the arid (AR), tropical (TR) and finally the high albedo (HA) zones. This representativeness of the climate zones is also useful to perceive the distribution of solar radiation measuring stations around the globe and how this may affect the model development. One can conclude that the high albedo and the tropical climate zones are not well represented. However, nowadays there are more meteorological stations in these climate zones (see, e.g. Ref. [69]). The most typical length of the training datasets is two years, followed by one, three and four years. It worths to note that some authors used twenty and even thirty years of data to derive their models (e.g. Refs. [27,61]).

\section{Model development and test data}

\subsection{Model formulation}

The model proposed in this study is based on the correlation of two limiting functions for large and small values of $K_{t}$ through the expression disclosed by Churchill and Usagi [70] for the correlation of transfer phenomena, described as follows:

$Y=\left(1+Z^{n}\right)^{\frac{1}{n}}$

where the arbitrary exponent $n$ needs to be selected in order to correlate those functions accurately [70]. This expression can be used to any phenomenon varying uniformly, for example in heat transfer modelling 
Table 1

Review of hourly and sub-hourly $K_{d}$ models whose only predictor is $K_{t}$.

\begin{tabular}{|c|c|c|c|c|c|c|}
\hline Model & Reference & Location & Climate & Data period & Constrains & $K_{d}$ \\
\hline 1 & $\begin{array}{l}\text { Orgill and } \\
\text { Hollands [15] }\end{array}$ & Toronto, Canada & $\mathrm{TM}$ & 1967-1971 & $\begin{array}{l}K_{t} \leq 0.35 \\
0.35 \leq K_{t} \leq 0.75 \\
K_{t}>0.75\end{array}$ & $\begin{array}{l}1.0-0.249 K_{t} \\
1.557-1.840 K_{t} \\
0.177\end{array}$ \\
\hline 2 & Bruno [18] & $\begin{array}{l}\text { Hamburg, } \\
\text { Germany }\end{array}$ & $\mathrm{TM}$ & $1973-1974$ & - & $0.310 K_{t}+0.139 \sin \left(4.620 K_{t}\right)$ \\
\hline 3 & Erbs et al. [19] & $\begin{array}{l}\text { Four cities in the } \\
\text { United States }\end{array}$ & $\begin{array}{l}\text { Various } \\
\text { climates }\end{array}$ & $\begin{array}{l}1974-1976 \\
\text { (Various data } \\
\text { periods) }\end{array}$ & $\begin{array}{l}K_{t} \leq 0.22 \\
0.22<K_{t} \leq 0.80 \\
K_{t}>0.80\end{array}$ & $\begin{array}{l}1.0-0.0900 K_{t} \\
0.9511-0.1604 K_{t}+4.3880 K_{t}^{2}-16.6380 K_{t}^{3}+12.3360 K_{t}^{4} \\
0.165\end{array}$ \\
\hline 4 & Spencer [28] & Albany, Australia & AR & 1973-1977 & $\begin{array}{l}K_{t}<0.35 \\
0.35 \leq K_{t} \leq 0.75 \\
K_{t}>0.75\end{array}$ & $\begin{array}{l}0.890 \\
1.414-1.736 K_{t} \\
0.110\end{array}$ \\
\hline 5 & Spencer [28] & $\begin{array}{l}\text { Alice Springs, } \\
\text { Australia }\end{array}$ & AR & 1974-1977 & $\begin{array}{l}K_{t}<0.35 \\
0.35 \leq K_{t} \leq 0.75 \\
K_{t}>0.75\end{array}$ & $\begin{array}{l}0.750 \\
1.183-1.444 K_{t} \\
0.110\end{array}$ \\
\hline 6 & Spencer [28] & $\begin{array}{l}\text { Geraldton, } \\
\text { Australia }\end{array}$ & $\mathrm{AR}$ & $1972-1977$ & $\begin{array}{l}K_{t}<0.35 \\
0.35 \leq K_{t} \leq 0.75 \\
K_{t}>0.75\end{array}$ & $\begin{array}{l}0.850 \\
1.345-1.644 K_{t} \\
0.110\end{array}$ \\
\hline 7 & Spencer [28] & $\begin{array}{l}\text { Guildford, } \\
\text { Australia }\end{array}$ & $\mathrm{AR}$ & 1975-1977 & $\begin{array}{l}K_{t}<0.35 \\
0.35 \leq K_{t} \leq 0.75 \\
K_{t}>0.75\end{array}$ & $\begin{array}{l}0.780 \\
1.254-1.595 K_{t} \\
0.060\end{array}$ \\
\hline 8 & Spencer [28] & Hobart, Australia & $\mathrm{TM}$ & 1971-1977 & $\begin{array}{l}K_{t}<0.35 \\
0.35 \leq K_{t} \leq 0.75 \\
K_{t}>0.75\end{array}$ & $\begin{array}{l}0.860 \\
1.360-1.678 K_{t} \\
0.100\end{array}$ \\
\hline 9 & Spencer [28] & $\begin{array}{l}\text { Laverton, } \\
\text { Australia }\end{array}$ & $\mathrm{AR}$ & 1976-1977 & $\begin{array}{l}K_{t}<0.35 \\
0.35 \leq K_{t} \leq 0.75 \\
K_{t}>0.75\end{array}$ & $\begin{array}{l}0.860 \\
1.360-1.678 K_{t} \\
0.150\end{array}$ \\
\hline 10 & Spencer [28] & $\begin{array}{l}\text { Melbourne, } \\
\text { Australia }\end{array}$ & $\mathrm{AR}$ & 1970-1977 & $\begin{array}{l}K_{t}<0.35 \\
0.35 \leq K_{t} \leq 0.75 \\
K_{t}>0.75\end{array}$ & $\begin{array}{l}0.850 \\
1.352-1.668 K_{t} \\
0.100\end{array}$ \\
\hline 11 & Spencer [28] & Mildura, Australia & $\mathrm{AR}$ & $1972-1977$ & $\begin{array}{l}K_{t}<0.35 \\
0.35 \leq K_{t} \leq 0.75 \\
K_{t}>0.75\end{array}$ & $\begin{array}{l}0.870 \\
1.366-1.666 K_{t} \\
0.120\end{array}$ \\
\hline 12 & Spencer [28] & $\begin{array}{l}\text { Mt Gambier, } \\
\text { Australia }\end{array}$ & $\mathrm{AR}$ & 1974-1977 & $\begin{array}{l}K_{t}<0.35 \\
0.35 \leq K_{t} \leq 0.75 \\
K_{t}>0.75\end{array}$ & $\begin{array}{l}0.930 \\
1.450-1.744 K_{t} \\
0.140\end{array}$ \\
\hline 13 & Spencer [28] & $\begin{array}{l}\text { Port Hedland, } \\
\text { Australia }\end{array}$ & $\mathrm{AR}$ & 1974-1977 & $\begin{array}{l}K_{t}<0.35 \\
0.35 \leq K_{t} \leq 0.75 \\
K_{t}>0.75\end{array}$ & $\begin{array}{l}0.710 \\
1.142-1.431 K_{t} \\
0.070\end{array}$ \\
\hline 14 & Spencer [28] & $\begin{array}{l}\text { Rockhampton, } \\
\text { Australia }\end{array}$ & $\mathrm{AR}$ & 1974-1977 & $\begin{array}{l}K_{t}<0.35 \\
0.35 \leq K_{t} \leq 0.75 \\
K_{t}>0.75\end{array}$ & $\begin{array}{l}0.790 \\
1.245-1.527 K_{t} \\
0.100\end{array}$ \\
\hline 15 & Spencer [28] & $\begin{array}{l}\text { Waga Waga, } \\
\text { Australia }\end{array}$ & $\mathrm{AR}$ & 1974-1977 & $\begin{array}{l}K_{t}<0.35 \\
0.35 \leq K_{t} \leq 0.75 \\
K_{t}>0.75\end{array}$ & $\begin{array}{l}0.800 \\
1.280-1.605 K_{t} \\
0.080\end{array}$ \\
\hline 16 & Spencer [28] & $\begin{array}{l}\text { Australia } \\
\text { (average) }\end{array}$ & $\begin{array}{l}\text { Various } \\
\text { climates }\end{array}$ & $\begin{array}{l}\text { 1970-1977 } \\
\text { (Various data } \\
\text { periods) }\end{array}$ & $\begin{array}{l}K_{t}<0.35 \\
0.35 \leq K_{t} \leq 0.75 \\
K_{t}>0.75\end{array}$ & $\begin{array}{l}0.830 \\
1.321-1.624 K_{t} \\
0.100\end{array}$ \\
\hline 17 & Hawlader [20] & Singapore & $\mathrm{TM}$ & 1962 & $\begin{array}{l}K_{t}<0.225 \\
0.225 \leq K_{t} \leq 0.775\end{array}$ & $\begin{array}{l}0.9150 \\
1.1389-0.9422 K_{t}-0.3878 K_{t}^{2}\end{array}$ \\
\hline 18 & Ineichen et al. [29] & $\begin{array}{l}\text { Geneva, } \\
\text { Switzerland }\end{array}$ & $\mathrm{TM}$ & 1978-1984 & $\begin{array}{l}K_{t}>0.775 \\
K_{t}<0.15 \\
K_{t} \geq 0.15\end{array}$ & $\begin{array}{l}0.2150 \\
0.98 \\
0.80+2.25 K_{t}-7.93 K_{t}^{2}+5.26 K_{t}^{3}\end{array}$ \\
\hline 19 & Ineichen et al. [29] & $\begin{array}{l}\text { Geneva, } \\
\text { Switzerland }\end{array}$ & $\mathrm{TM}$ & 1978-1984 & $\begin{array}{l}K_{t}<0.25 \\
0.25 \leq K_{t} \leq 0.80 \\
K_{t}>0.80\end{array}$ & $\begin{array}{l}1.0 \\
1.38-1.52 K_{t} \\
0.16\end{array}$ \\
\hline 20 & Ineichen et al. [29] & $\begin{array}{l}\text { Geneva, } \\
\text { Switzerland }\end{array}$ & $\mathrm{TM}$ & 1978-1984 & $\begin{array}{l}K_{t}<0.25 \\
K_{t} \geq 0.25\end{array}$ & $\begin{array}{l}1.0 \\
1.28 K_{t}-1.40 K_{t}^{2}\end{array}$ \\
\hline 21 & Muneer et al. [21] & New Delhi, India & $\mathrm{TR}$ & 1971,1974 & $\begin{array}{l}K_{t}<0.175 \\
0.175 \leq K_{t} \leq 0.775 \\
K_{t}>0.775\end{array}$ & $\begin{array}{l}0.9500 \\
0.9698+0.4353 K_{t}-3.4499 K_{t}^{2}+2.1888 K_{t}^{3} \\
0.2600\end{array}$ \\
\hline 22 & Bakhsh et al. [30] & $\begin{array}{l}\text { Dharan, Saudi } \\
\text { Arabia }\end{array}$ & $\mathrm{AR}$ & 1983-1984 & $\begin{array}{l}K_{t}<0.23 \\
0.23 \leq K_{t} \leq 0.80 \\
K_{t}>0.80\end{array}$ & $\begin{array}{l}1.0-0.220 K_{t} \\
1.235-1.260 K_{t} \\
0.225\end{array}$ \\
\hline 23 & Hollands [31] & Toronto, Canada & TM & $1967-1971$ & - & $\begin{array}{l}{\left[1-b-\sqrt{(1-b)^{2}-4 a b^{2} K_{t}\left(1-a K_{t}\right)}\right] /\left(2 a b K_{t}\right)} \\
a=1.115 ; b=0.491\end{array}$ \\
\hline 24 & Reindl et al. [6] & $\begin{array}{l}\text { Five locations in } \\
\text { North America } \\
\text { and Europe }\end{array}$ & $\begin{array}{l}\text { Various } \\
\text { climates }\end{array}$ & $\begin{array}{l}\text { 1979-1982 } \\
\text { (Various data } \\
\text { periods) }\end{array}$ & $\begin{array}{l}K_{t} \leq 0.30 \\
0.30<K_{t}<0.78 \\
K_{t} \geq 0.78\end{array}$ & $\begin{array}{l}1.020-0.248 K_{t} \\
1.450-1.670 K_{t} \\
0.147\end{array}$ \\
\hline 25 & Al-Rihai [32] & Fudhaliyah, Iraq & $\mathrm{AR}$ & $1984-1987$ & $\begin{array}{l}K_{t}<0.25 \\
0.25 \leq K_{t} \leq 0.70 \\
K_{t}>0.70\end{array}$ & $\begin{array}{l}0.932 \\
1.293-1.631 K_{t} \\
0.151\end{array}$ \\
\hline
\end{tabular}


Table 1 (continued)

\begin{tabular}{|c|c|c|c|c|c|c|}
\hline Model & Reference & Location & Climate & Data period & Constrains & $K_{d}$ \\
\hline 26 & Bourges [33] & $\begin{array}{l}37 \text { stations across } \\
\text { Europe }\end{array}$ & $\mathrm{TM}$ & $\begin{array}{l}\text { At least four } \\
\text { years of } \\
\text { measurements }\end{array}$ & $\begin{array}{l}K_{t} \leq 0.20 \\
0.20<K_{t} \leq 0.35 \\
0.35<K_{t} \leq 0.75 \\
K_{t}>0.75\end{array}$ & $\begin{array}{l}1.0 \\
1.116-0.580 K_{t} \\
1.557-1.840 K_{t} \\
0.177\end{array}$ \\
\hline 27 & $\begin{array}{l}\text { Chandrasekaran } \\
\text { and Kumar [34] }\end{array}$ & Madras, India & TR & 1983-1987 & $\begin{array}{l}K_{t} \leq 0.24 \\
0.24<K_{t} \leq 0.80 \\
K_{t}>0.80\end{array}$ & $\begin{array}{l}1.0086-0.1780 K_{t} \\
0.9686+0.1325 K_{t}+1.4183 K_{t}^{2}-10.1860 K_{t}^{3}+8.3733 K_{t}^{4} \\
0.1970\end{array}$ \\
\hline 28 & $\begin{array}{l}\text { Chendo and } \\
\text { Maduekwe [35] }\end{array}$ & Lagos, Nigeria & $\mathrm{TM}$ & $\begin{array}{l}\text { Two years of } \\
\text { measurements }\end{array}$ & $\begin{array}{l}K_{t} \leq 0.30 \\
0.30<K_{t}<0.80 \\
K_{t} \geq 0.80\end{array}$ & $\begin{array}{l}1.022-0.156 K_{t} \\
1.385-1.396 K_{t} \\
0.264\end{array}$ \\
\hline 29 & $\begin{array}{l}\text { Maduekwe and } \\
\text { Chendo [36] }\end{array}$ & Lagos, Nigeria & $\mathrm{TM}$ & 1990-1991 & $\begin{array}{l}K_{t} \leq 0.30 \\
0.30<K_{t}<0.80 \\
K_{t} \geq 0.80\end{array}$ & $\begin{array}{l}1.021-0.151 K_{t} \\
1.385-1.396 K_{t} \\
0.295\end{array}$ \\
\hline 30 & Lam and Li [37] & Hong Kong, China & TM & 1991-1994 & $\begin{array}{l}K_{t} \leq 0.15 \\
0.15<K_{t} \leq 0.70 \\
K_{t}>0.70\end{array}$ & $\begin{array}{l}0.977 \\
1.237-1.361 K_{t} \\
0.273\end{array}$ \\
\hline 31 & Hijazin [38] & Amman, Jordan & $\mathrm{AR}$ & 1985 & $\begin{array}{l}K_{t}<0.10 \\
0.10 \leq K_{t} \leq 0.80 \\
K_{t}>0.80\end{array}$ & $\begin{array}{l}0.744 \\
0.842-0.977 K_{t} \\
0.060\end{array}$ \\
\hline 32 & Hijazin [38] & Amman, Jordan & AR & 1985 & - & $0.847-0.985 K_{t}$ \\
\hline 33 & $\begin{array}{l}\text { González and } \\
\text { Calbó [39] }\end{array}$ & $\begin{array}{l}\text { Two locations in } \\
\text { Iberian Peninsula }\end{array}$ & $\mathrm{TM}$ & $\begin{array}{l}\text { 1994-1996 } \\
\text { (Various data } \\
\text { periods) }\end{array}$ & $\begin{array}{l}0.25<K_{t}<0.75 \\
K_{t} \geq 0.75\end{array}$ & $\begin{array}{l}1.421-1.670 K_{t} \\
-0.043+0.290 K_{t}\end{array}$ \\
\hline 34 & Boland et al. [26] & Geelong, Australia & TM & 67 days & - & $1.0 /\left[1.0+\exp \left\{8.645\left(K_{t}-0.613\right)\right\}\right]$ \\
\hline 35 & Boland et al. [26] & Geelong, Australia & TM & 67 days & - & $1.0 /\left[1.0+\exp \left\{7.997\left(K_{t}-0.586\right)\right\}\right]$ \\
\hline 36 & $\begin{array}{l}\text { De Miguel et al. } \\
\text { [40] }\end{array}$ & $\begin{array}{l}\text { North } \\
\text { Mediterranean } \\
\text { belt area (11 } \\
\text { stations) }\end{array}$ & $\mathrm{TM}$ & $\begin{array}{l}\text { 1974-1996 } \\
\text { (Various data } \\
\text { periods) }\end{array}$ & $\begin{array}{l}K_{t} \leq 0.21 \\
0.21<K_{t} \leq 0.76 \\
K_{t}>0.76\end{array}$ & $\begin{array}{l}0.995-0.081 K_{t} \\
0.724+2.738 K_{t}-8.320 K_{t}^{2}+4.967 K_{t}^{3} \\
0.180\end{array}$ \\
\hline 37 & Li and Lam [41] & Hong Kong, China & TM & 1991-1998 & $\begin{array}{l}K_{t} \leq 0.15 \\
0.15<K_{t} \leq 0.70 \\
K_{t}>0.70\end{array}$ & $\begin{array}{l}0.976 \\
0.996+0.036 K_{t}-1.589 K_{t}^{2} \\
0.230\end{array}$ \\
\hline 38 & Oliveira et al. [42] & São Paulo, Brazil & $\mathrm{TM}$ & 1994-1999 & $0.17<K_{t}<0.75$ & $0.97+0.80 K_{t}-3.00 K_{t}^{2}-3.1 K_{t}^{3}+5.2 K_{t}^{4}$ \\
\hline 39 & $\begin{array}{l}\text { Ulgen and } \\
\text { Hepbasli [43] }\end{array}$ & Izmir, Turkey & $\mathrm{TM}$ & 1994-1998 & $\begin{array}{l}K_{t} \leq 0.32 \\
0.32<K_{t} \leq 0.62 \\
K_{t}>0.62\end{array}$ & $\begin{array}{l}0.6800 \\
1.0609-1.2138 K_{t} \\
0.3000\end{array}$ \\
\hline 40 & $\begin{array}{l}\text { Ulgen and } \\
\text { Hepbasli [43] }\end{array}$ & Izmir, Turkey & TM & 1994-1998 & $\begin{array}{l}K_{t} \leq 0.32 \\
0.32<K_{t} \leq 0.62 \\
K_{t}>0.62\end{array}$ & $\begin{array}{l}0.6800 \\
0.0743-19.3430 K_{t}+206.9100 K_{t}^{2}-719.7200 K_{t}^{3}+1053.4000 K_{t}^{4}-562.69 K_{t}^{5} \\
0.3000\end{array}$ \\
\hline 41 & $\begin{array}{l}\text { Karatasou et al. } \\
\text { [44] }\end{array}$ & Athens, Greece & TM & 1996-1998 & $\begin{array}{l}K_{t} \leq 0.78 \\
K_{t}>0.78\end{array}$ & $\begin{array}{l}0.9995-0.0500 K_{t}-2.4156 K_{t}^{2}+1.4926 K_{t}^{3} \\
0.2000\end{array}$ \\
\hline 42 & $\begin{array}{l}\text { Tsubo and Walker } \\
\text { [45] }\end{array}$ & Southern Africa & $\mathrm{AR}$ & 2000 & - & $0.613-0.334 K_{t}+0.121 K_{t}^{2}$ \\
\hline 43 & $\begin{array}{l}\text { Tsubo and Walker } \\
\text { [45] }\end{array}$ & Southern Africa & $\mathrm{AR}$ & 2000 & $\begin{array}{l}K_{t}<0.140 \\
0.140 \leq K_{t} \leq 0.794 \\
K_{t}>0.794\end{array}$ & 0.907 \\
\hline 44 & $\begin{array}{l}\text { Tsubo and Walker } \\
\text { [45] }\end{array}$ & Southern Africa & $\mathrm{AR}$ & 2000 & $\begin{array}{l}K_{t}<0.140 \\
0.140 \leq K_{t} \leq 0.794 \\
K_{t}>0.793\end{array}$ & $\begin{array}{l}0.907 \\
1.063-1.114 K_{t} \\
0.180\end{array}$ \\
\hline 45 & Soares et al. [46] & São Paulo, Brazil & $\mathrm{TM}$ & $1998-2001$ & - & $0.90+1.10 K_{t}-4.50 K_{t}^{2}+0.01 K_{t}^{3}+3.14 K_{t}^{4}$ \\
\hline 46 & Mondol et al. [47] & $\begin{array}{l}\text { Ballymena, } \\
\text { Northern Ireland }\end{array}$ & $\mathrm{TM}$ & $\begin{array}{l}21 \text { months of } \\
\text { data }\end{array}$ & $\begin{array}{l}K_{t} \leq 0.20 \\
K_{t}>0.20\end{array}$ & $\begin{array}{l}0.9800 \\
0.5836+3.6259 K_{t}-10.1710 K_{t}^{2}+6.3380 K_{t}^{3}\end{array}$ \\
\hline 47 & $\begin{array}{l}\text { Jacovides et al. } \\
\text { [48] }\end{array}$ & Athalassa, Cyprus & $\mathrm{AR}$ & 1998-2002 & $\begin{array}{l}K_{t} \leq 0.10 \\
0.10<K_{t} \leq 0.80 \\
K_{t}>0.80\end{array}$ & $\begin{array}{l}0.987 \\
0.940+0.937 K_{t}-5.010 K_{t}^{2}+3.320 K_{t}^{3} \\
0.177\end{array}$ \\
\hline 48 & Elminir et al. [49] & Aswan, Egypt & $\mathrm{AR}$ & 1999-2001 & $\begin{array}{l}K_{t} \leq 0.22 \\
0.22<K_{t} \leq 0.80\end{array}$ & $\begin{array}{l}0.653-1.728 K_{t} \\
0.724-1.821 K_{t}+8.221 K_{t}^{2}-16.370 K_{t}^{3}+9.845 K_{t}^{4}\end{array}$ \\
\hline 49 & Elminir et al. [49] & Cairo, Egypt & $\mathrm{AR}$ & 2003 & $\begin{array}{l}K_{t}>0.80 \\
K_{t} \leq 0.22 \\
0.22<K_{t} \leq 0.80 \\
K_{t}>0.80\end{array}$ & $\begin{array}{l}0.217 \\
0.793-2.198 K_{t} \\
1.341-9.566 K_{t}+32.200 K_{t}^{2}-47.909 K_{t}^{3}+25.419 K_{t}^{4} \\
0.131\end{array}$ \\
\hline 50 & Elminir et al. [49] & $\begin{array}{l}\text { South-Valley, } \\
\text { Egypt }\end{array}$ & $\mathrm{AR}$ & 2003 & $\begin{array}{l}K_{t} \leq 0.22 \\
0.22<K_{t} \leq 0.80 \\
K_{t}>0.80\end{array}$ & $\begin{array}{l}0.8526-1.7780 K_{t} \\
0.8140-1.1060 K_{t}+0.3660 K_{t}^{2}-0.9970 K_{t}^{3}+1.2210 K_{t}^{4} \\
0.213\end{array}$ \\
\hline 51 & Boland et al. [50] & $\begin{array}{l}\text { Adelaide, } \\
\text { Australia }\end{array}$ & $\mathrm{AR}$ & - & - & $1.0 /\left[1.0+\exp \left(-5.83+9.87 K_{t}\right)\right]$ \\
\hline 52 & Boland et al. [50] & $\begin{array}{l}\text { Bracknell, } \\
\text { England }\end{array}$ & $\mathrm{TM}$ & - & - & $1.0 /\left[1.0+\exp \left(-4.38+6.62 K_{t}\right)\right]$ \\
\hline 53 & Boland et al. [50] & Darwin, Australia & TM & - & - & $1.0 /\left[1.0+\exp \left(-4.53+8.05 K_{t}\right)\right]$ \\
\hline 54 & Boland et al. [50] & Lisbon, Portugal & TM & - & - & $1.0 /\left[1.0+\exp \left(-4.80+7.98 K_{t}\right)\right]$ \\
\hline 55 & Boland et al. [50] & Macau, China & $\mathrm{TM}$ & - & - & $1.0 /\left[1.0+\exp \left(-4.87+8.12 K_{t}\right)\right]$ \\
\hline
\end{tabular}


Table 1 (continued)

\begin{tabular}{|c|c|c|c|c|c|c|}
\hline Model & Reference & Location & Climate & Data period & Constrains & $K_{d}$ \\
\hline 56 & Boland et al. [50] & $\begin{array}{l}\text { Maputo, } \\
\text { Mozambique }\end{array}$ & $\mathrm{AR}$ & - & - & $1.0 /\left[1.0+\exp \left(-5.18+8.80 K_{t}\right)\right]$ \\
\hline 57 & Boland et al. [50] & Uccle, Belgium & TM & - & - & $1.0 /\left[1.0+\exp \left(-4.94+8.66 K_{t}\right)\right]$ \\
\hline 58 & Boland et al. [50] & $\begin{array}{l}\text { Multi-location } \\
\text { average }\end{array}$ & $\begin{array}{l}\text { Various } \\
\text { climates }\end{array}$ & - & - & $1.0 /\left[1.0+\exp \left(-4.94+8.30 K_{t}\right)\right]$ \\
\hline 59 & $\begin{array}{l}\text { Boland and Ridley } \\
\text { [51] }\end{array}$ & $\begin{array}{l}\text { Multi-locations } \\
\text { worldwide }\end{array}$ & $\begin{array}{l}\text { Various } \\
\text { climates }\end{array}$ & - & - & $1.0 /\left[1.0+\exp \left(-5.00+8.60 K_{t}\right)\right]$ \\
\hline 60 & $\begin{array}{l}\text { Furlan and } \\
\text { Oliveira [52] }\end{array}$ & Sâo Paulo, Brazil & $\mathrm{TM}$ & 2002 & $\begin{array}{l}K_{t} \leq 0.228 \\
K_{t}>0.228\end{array}$ & $\begin{array}{l}0.961 \\
1.337-1.650 K_{t}\end{array}$ \\
\hline 61 & Mondol et al. [53] & $\begin{array}{l}\text { Aldergrove, } \\
\text { Northern Irland }\end{array}$ & TM & 1989-1998 & $\begin{array}{l}K_{t} \leq 0.20 \\
0.20<K_{t} \leq 0.70 \\
K_{t}>0.70\end{array}$ & $\begin{array}{l}0.9800 \\
0.6109+3.6259 K_{t}-10.1710 K_{t}^{2}+6.3380 K_{t}^{3} \\
0.6720-0.4740 K_{t}\end{array}$ \\
\hline 62 & Moreno et al. [54] & Seville, Spain & TM & $2000-2008$ & $\begin{array}{l}K_{t} \leq 0.27 \\
0.27<K_{t} \leq 0.82 \\
K_{t}>0.82\end{array}$ & $\begin{array}{l}0.9930 \\
1.4946-1.7899 K_{t} \\
0.0450\end{array}$ \\
\hline 63 & Pagola et al. [55] & $\begin{array}{l}3 \text { locations in } \\
\text { Spain }\end{array}$ & TM & $2005-2008$ & $\begin{array}{l}K_{t} \leq 0.35 \\
0.35<K_{t} \leq 0.75 \\
K_{t}>0.75\end{array}$ & $\begin{array}{l}0.9818-0.5870 K_{t} \\
1.2056-1.3240 K_{t} \\
0.2552\end{array}$ \\
\hline 64 & Pagola et al. [55] & $\begin{array}{l}3 \text { locations in } \\
\text { Spain }\end{array}$ & TM & $2005-2008$ & $\begin{array}{l}K_{t} \leq 0.22 \\
0.22<K_{t} \leq 0.80 \\
K_{t}>0.80\end{array}$ & $\begin{array}{l}0.9522-0.3119 K_{t} \\
0.6059+2.9877 K_{t}-10.5675 K_{t}^{2}+10.1833 K_{t}^{3}-3.0475 K_{t}^{4} \\
0.3209\end{array}$ \\
\hline 65 & $\begin{array}{l}\text { Posadillo and } \\
\text { Lopez Luque [56] }\end{array}$ & Córdoba, Spain & TM & 1993-2002 & - & $K_{t}\left(1.17-1.381 K_{t}\right)$ \\
\hline 66 & $\begin{array}{l}\text { Posadillo and } \\
\text { Lopez Luque [56] }\end{array}$ & Córdoba, Spain & $\mathrm{TM}$ & 1993-2002 & - & $-0.00829+1.16300 K_{t}+0.43300 K_{t}^{2}-5.83900 K_{t}^{3}+4.64880 K_{t}^{4}$ \\
\hline 67 & Janjai et al. [57] & $\begin{array}{l}\text { Chiang Mai, } \\
\text { Thailand }\end{array}$ & TR & 1995-2006 & - & $0.9429-0.3707 K_{t}+6.4927 K_{t}^{2}-30.3560 K_{t}^{3}+39.1626 K_{t}^{4}-15.4850 K_{t}^{5}$ \\
\hline 68 & Janjai et al. [57] & $\begin{array}{l}\text { Nakhon Pathom, } \\
\text { Thailand }\end{array}$ & TR & 1995-2006 & - & $0.7699+2.3552 K_{t}-8.1480 K_{t}^{2}+5.3811 K_{t}^{3}$ \\
\hline 69 & Janjai et al. [57] & $\begin{array}{l}\text { Songkhla, } \\
\text { Thailand }\end{array}$ & TR & 1995-2006 & - & $0.869+1.559 K_{t}-11.176 K_{t}^{2}+26.143 K_{t}^{3}-38.302 K_{t}^{4}+31.799 K_{t}^{5}-10.602 K_{t}^{6}$ \\
\hline 70 & Janjai et al. [57] & $\begin{array}{l}\text { Ubon Ratchathani, } \\
\text { Thailand }\end{array}$ & TR & 1995-2006 & - & $0.846+1.841 K_{t}-13.425 K_{t}^{2}+42.888 K_{t}^{3}-85.804 K_{t}^{4}+84.476 K_{t}^{5}-30.637 K_{t}^{6}$ \\
\hline 71 & $\begin{array}{l}\text { Ruiz-Arias et al. } \\
{[27]}\end{array}$ & Albacete, Spain & $\mathrm{TM}$ & 2002-2006 & - & $0.086+0.880 \mathrm{e}^{-\exp \left(-3.877+6.138 K_{t}\right)}$ \\
\hline 72 & $\begin{array}{l}\text { Ruiz-Arias et al. } \\
\text { [27] }\end{array}$ & Boulder, USA & TM & $1961-1990$ & - & $0.967-1.024 \mathrm{e}^{-\exp \left(2.473-5.324 K_{t}\right)}$ \\
\hline 73 & $\begin{array}{l}\text { Ruiz-Arias et al. } \\
\text { [27] }\end{array}$ & Dresden, Germany & $\mathrm{TM}$ & $1981-1990$ & - & $0.140+0.962 \mathrm{e}^{-\exp \left(-1.976+4.067 K_{t}\right)}$ \\
\hline 74 & $\begin{array}{l}\text { Ruiz-Arias et al. } \\
\text { [27] }\end{array}$ & Pittsburgh, USA & TM & $1961-1990$ & - & $1.001-1.000 \mathrm{e}^{-\exp (2.450-5.048 K t)}$ \\
\hline 75 & $\begin{array}{l}\text { Ruiz-Arias et al. } \\
\text { [27] }\end{array}$ & Savannah, USA & TM & $1961-1990$ & - & $0.988-1.000 \mathrm{e}^{-\exp \left(2.456-5.172 K_{t}\right)}$ \\
\hline 76 & $\begin{array}{l}\text { Ruiz-Arias et al. } \\
{[27]}\end{array}$ & Talkeetna, USA & HA & $1961-1990$ & - & $0.985-0.962 \mathrm{e}^{-\exp \left(2.655-6.003 K_{t}\right)}$ \\
\hline 77 & $\begin{array}{l}\text { Ruiz-Arias et al. } \\
\text { [27] }\end{array}$ & Tucson, USA & $\mathrm{AR}$ & 1961-1990 & - & $0.988-1.073 \mathrm{e}^{-\exp \left(2.298-4.909 K_{t}\right)}$ \\
\hline 78 & $\begin{array}{l}\text { Ruiz-Arias et al. } \\
\text { [27] }\end{array}$ & $\begin{array}{l}7 \text { locations in } \\
\text { Europe and USA }\end{array}$ & $\begin{array}{l}\text { Various } \\
\text { climates }\end{array}$ & $\begin{array}{l}1961-2006 \\
\text { (Various data } \\
\text { periods) }\end{array}$ & - & $0.952-1.041 \mathrm{e}^{-\exp (2.3-4.702 K t)}$ \\
\hline 79 & Torres et al. [58] & Pamplona, Spain & TM & $2006-2008$ & $\begin{array}{l}K_{t} \leq 0.24 \\
0.24<K_{t}<0.75 \\
K_{t} \geq 0.75\end{array}$ & $\begin{array}{l}1.0058-0.2195 K_{t} \\
1.3264-1.5120 K_{t} \\
0.1923\end{array}$ \\
\hline 80 & Torres et al. [58] & Pamplona, Spain & TM & $2006-2008$ & $\begin{array}{l}K_{t} \leq 0.22 \\
0.22<K_{t}<0.75\end{array}$ & $\begin{array}{l}0.9920-0.0980 K_{t} \\
1.2158-1.0467 K_{t}-0.4480 K_{t}^{2}\end{array}$ \\
\hline 81 & Torres et al. [58] & Pamplona, Spain & TM & 2006-2008 & $\begin{array}{l}K_{t} \geq 0.75 \\
K_{t} \leq 0.22 \\
0.24<K_{t} \leq 0.755 \\
K_{t}>0.755\end{array}$ & $\begin{array}{l}0.1787 \\
0.9923-0.0980 K_{t} \\
1.1459-0.5612 K_{t}-1.4952 K_{t}^{2}+0.7103 K_{t}^{3} \\
0.1755\end{array}$ \\
\hline 82 & Torres et al. [58] & Pamplona, Spain & TM & $2006-2008$ & $\begin{array}{l}K_{t} \leq 0.225 \\
0.225<K_{t} \leq 0.755\end{array}$ & $\begin{array}{l}0.9943-0.1165 K_{t} \\
1.4101-2.9918 K_{t}+6.4599 K_{t}^{2}-10.3290 K_{t}^{3}+5.5140 K_{t}^{4}\end{array}$ \\
\hline 83 & Chikh et al. [59] & Alger, Algeria & $\mathrm{AR}$ & 1992 & $\begin{array}{l}K_{t}>0.755 \\
K_{t} \leq 0.175 \\
0.175<K_{t} \leq 0.87 \\
K_{t}>0.87\end{array}$ & $\begin{array}{l}0.1800 \\
1.0-0.232 K_{t} \\
1.170-1.230 K_{t} \\
0.203\end{array}$ \\
\hline 84 & Chikh et al. [59] & Bechar, Algeria & $\mathrm{AR}$ & 1990-1992 & $\begin{array}{l}K_{t} \leq 0.175 \\
0.175<K_{t} \leq 0.87 \\
K_{t}>0.87\end{array}$ & $\begin{array}{l}1.0-0.3000 K_{t} \\
1.1370-1.0770 K_{t} \\
0.2043\end{array}$ \\
\hline 85 & Chikh et al. [59] & $\begin{array}{l}\text { Tamanrasset, } \\
\text { Algeria }\end{array}$ & AR & 1990-1992 & $\begin{array}{l}K_{t} \leq 0.175 \\
0.175<K_{t} \leq 0.87 \\
K_{t}>0.87\end{array}$ & $\begin{array}{l}1.0-0.640 K_{t} \\
1.052-0.935 K_{t} \\
0.240\end{array}$ \\
\hline 86 & Sanchez et al. [60] & Badajoz, Spain & TM & 2009-2010 & $\begin{array}{l}K_{t}<0.30 \\
0.30 \leq K_{t} \leq 0.75 \\
K_{t}>0.75\end{array}$ & $\begin{array}{l}0.78 \\
1.23-1.43 K_{t} \\
0.13\end{array}$ \\
\hline
\end{tabular}


Table 1 (continued)

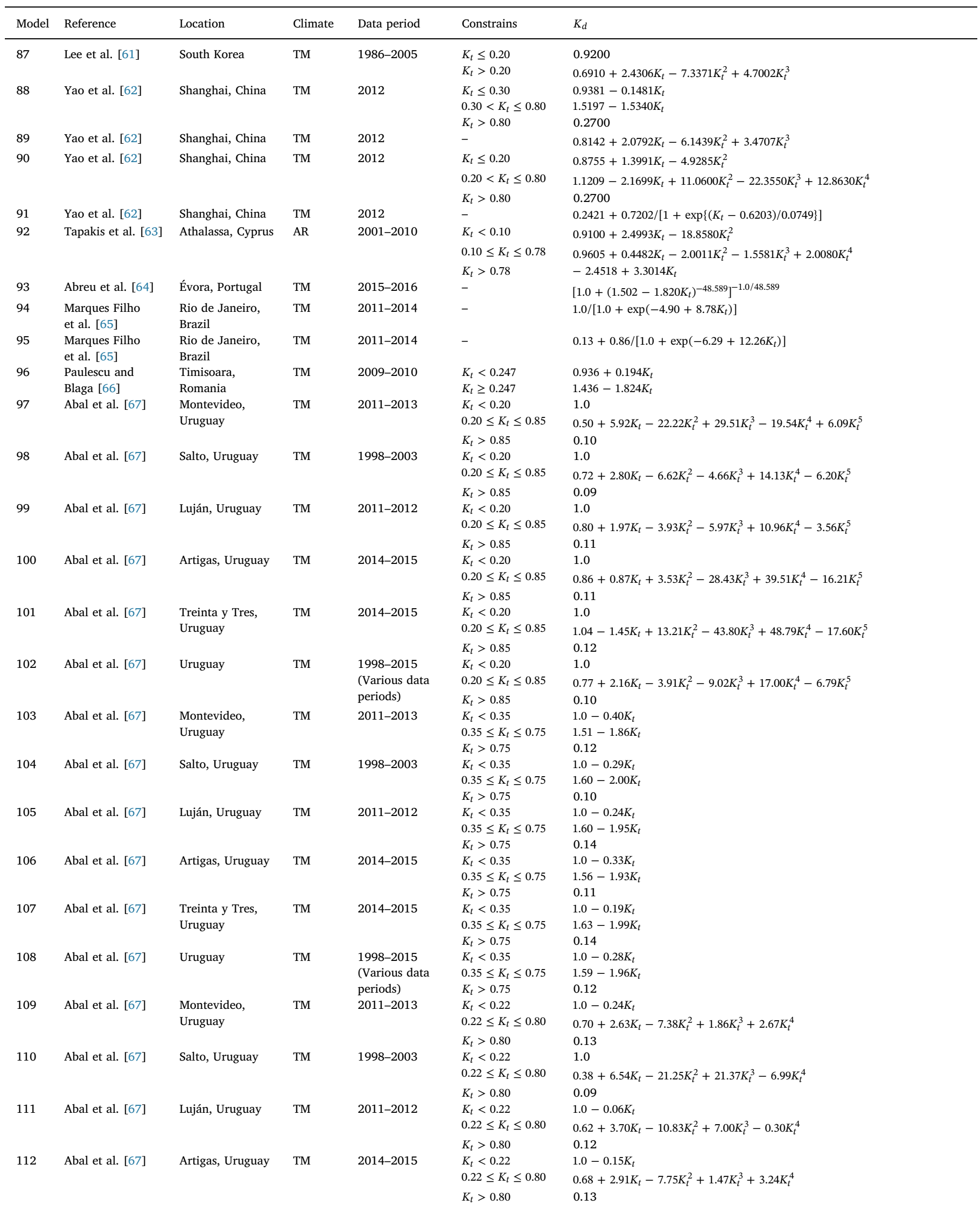


Table 1 (continued)

\begin{tabular}{|c|c|c|c|c|c|c|}
\hline Model & Reference & Location & Climate & Data period & Constrains & $K_{d}$ \\
\hline 113 & Abal et al. [67] & $\begin{array}{l}\text { Treinta y Tres, } \\
\text { Uruguay }\end{array}$ & TM & 2014-2015 & $\begin{array}{l}K_{t}<0.22 \\
0.22 \leq K_{t} \leq 0.80 \\
K_{t}>0.80\end{array}$ & $\begin{array}{l}1.0-0.10 K_{t} \\
0.85+0.98 K_{t}-0.06 K_{t}^{2}-9.75 K_{t}^{3}+8.62 K_{t}^{4} \\
0.13\end{array}$ \\
\hline 114 & Abal et al. [67] & Uruguay & $\mathrm{TM}$ & $\begin{array}{l}1998-2015 \\
\text { (Various data } \\
\text { periods) }\end{array}$ & $\begin{array}{l}K_{t}<0.22 \\
0.22 \leq K_{t} \leq 0.80 \\
K_{t}>0.80\end{array}$ & $\begin{array}{l}1.0-0.09 K_{t} \\
0.60+3.97 K_{t}-11.74 K_{t}^{2}+7.76 K_{t}^{3}-0.28 K_{t}^{4} \\
0.11\end{array}$ \\
\hline 115 & Abal et al. [67] & $\begin{array}{l}\text { Montevideo, } \\
\text { Uruguay }\end{array}$ & $\mathrm{TM}$ & $2011-2013$ & - & $0.95-0.97 \mathrm{e}^{-\exp \left(2.96-6.07 K_{t}\right)}$ \\
\hline 116 & Abal et al. [67] & Salto, Uruguay & $\mathrm{TM}$ & $1998-2003$ & - & $1.00-1.07 \mathrm{e}^{-\exp \left(2.82-5.82 K_{t}\right)}$ \\
\hline 117 & Abal et al. [67] & Luján, Uruguay & $\mathrm{TM}$ & $2011-2012$ & - & $0.98-1.05 \mathrm{e}^{-\exp \left(2.96-5.75 K_{t}\right)}$ \\
\hline 118 & Abal et al. [67] & Artigas, Uruguay & $\mathrm{TM}$ & 2014-2015 & - & $0.95-0.92 \mathrm{e}^{-\exp \left(3.57-7.32 K_{t}\right)}$ \\
\hline 119 & Abal et al. [67] & $\begin{array}{l}\text { Treinta y Tres, } \\
\text { Uruguay }\end{array}$ & TM & 2014-2015 & - & $0.96-0.97 \mathrm{e}^{-\exp \left(3.46-6.68 K_{t}\right)}$ \\
\hline 120 & Abal et al. [67] & Uruguay & TM & $\begin{array}{l}\text { 1998-2015 } \\
\text { (Various data } \\
\text { periods) }\end{array}$ & - & $0.97-1.01 \mathrm{e}^{-\exp \left(3.07-6.17 K_{t}\right)}$ \\
\hline 121 & $\begin{array}{l}\text { Al-Najar and Al- } \\
\text { Khazzar [68] }\end{array}$ & Baghdad, Iraq & $\mathrm{AR}$ & 2015 & - & $1.5973-4.6603 K_{t}+5.7190 K_{t}^{2}-2.5719 K_{t}^{3}$ \\
\hline
\end{tabular}

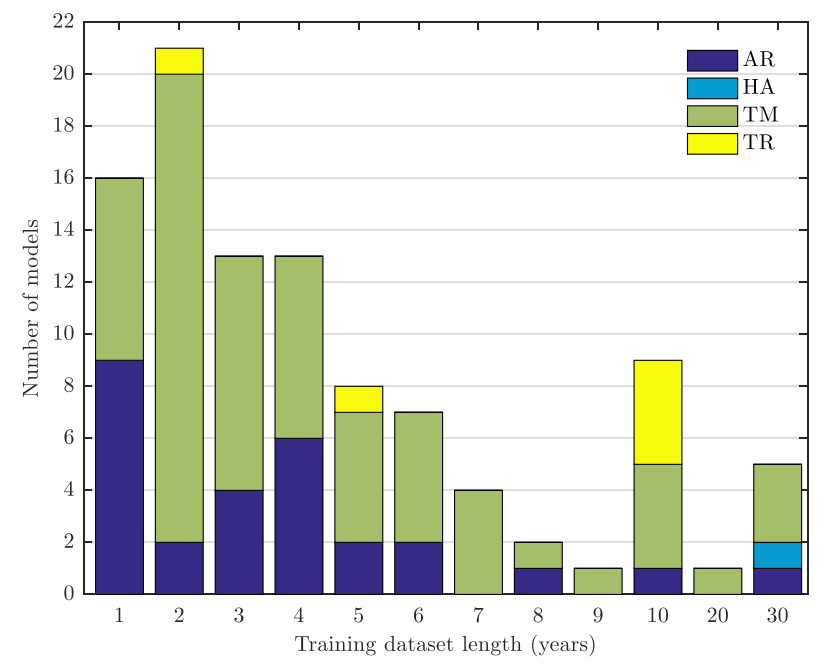

Fig. 1. Distribution of the models according to the length of the training datasets and climate zone: temperate (TM), arid (AR), tropical (TR) and high albedo (HA).

[71] and fluid flow and heat transfer optimisation when combined with the concept of the intersection of asymptotes [72,73]. In the case of modelling the diffuse fraction as a function of the clearness index, the functions used here are the physically possible limit of $K_{d}=1$ and a function $Z$ that is the best fit for the clear sky periods. The occurrence of cloud enhancement effects in 1-min resolution data is quite frequent $[1,14]$, then function $Z$ was defined as the best fit to the clear sky data using a second degree polynomial in the form:

$Z=A\left(K_{t}-0.5\right)^{2}+B\left(K_{t}-0.5\right)+1$

Since $K_{d}$ is a concave function of $K_{t}$, the exponents used in Eq. (1) must be $-n$ and $-1 / n$, and thus, the final form of the model is given by:

$K_{d}=\left\{1+\left[A\left(K_{t}-0.5\right)^{2}+B\left(K_{t}-0.5\right)+1\right]^{-n}\right\}^{-\frac{1}{n}}$

Fig. 2 shows the fitting of the model to the data of Fort Peck station (FPE), USA, as an example of the procedures implemented in this work for all the analysed stations. Red lines represent the limiting functions $K_{d}=1$ and $Z$ (fitted to the FPE dataset), and the blue line represents the fitted model. The three parameters of the adjusted model for FPE station are also presented, as well as the period of data used.

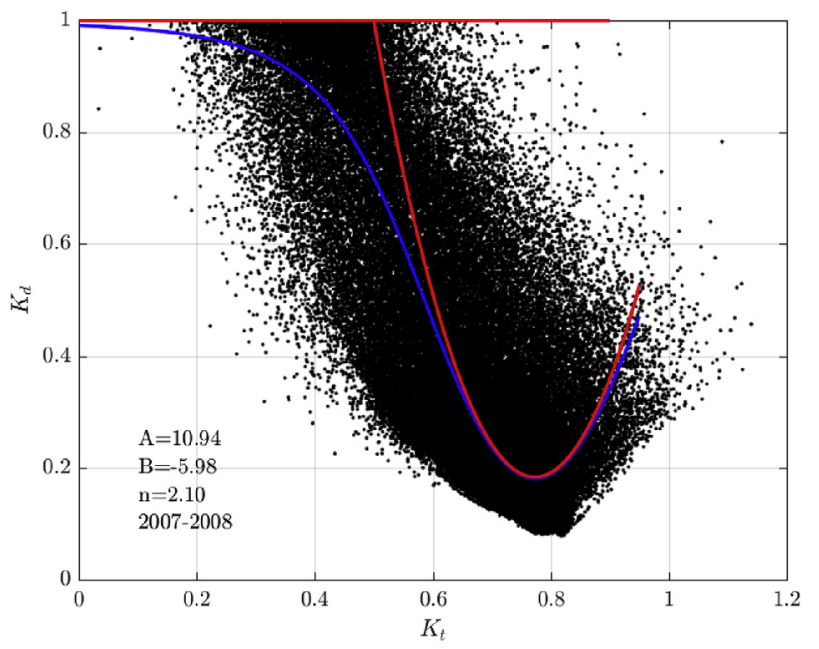

Fig. 2. Data for the FPE station (Fort Peck, USA) and representation of the limiting functions (red) and model correlation (blue). (For interpretation of the references to colour in this figure legend, the reader is referred to the Web version of this article.)

\subsection{Test stations and data quality control}

The data used in this study is from the Baseline Surface Radiation Network (BSRN) $[69,74]$ and the Institute of Earth Sciences (IES) at the University of Évora, Portugal. The BSRN is a project of the Global Energy and Water Cycle Experiment (GEWEX) under the umbrella of the World Climate Research Programme (WCRP). The primary objective of this network is to detect changes in the radiation field at the Earth's surface which may be related to climate changes. Measurements of solar radiation in the IES station are taken likewise as in the BSRN stations: the diffuse horizontal irradiation (DHI) is measured by a Kipp\& Zonnen CM6B pyranometer and shading ball attached to the sun tracker and the global horizontal irradiance is also measured by a Kipp\& Zonnen CM6B pyranometer. The sensors are installed on a Kipp\& Zonnen Solys2 sun tracker and are properly maintained and calibrated according to the BSRN and WMO guidelines and recommendations $[69,75]$. Table 2 shows detailed information on the stations used in this study: location, climate zone, data period, number of valid data points and mean GHI of all valid measurements.

Data quality control was performed by applying the quality filters defined by Long and Shi [76] for the global horizontal irradiance (GHI). Furthermore, $K_{d}$ values higher than 1 and lower than 0 were removed 
Table 2

Information on the data of BSRN and IES stations. Acronyms: AR (Arid), HA (High albedo), TM (Temperate), and TR (Tropical).

\begin{tabular}{|c|c|c|c|c|c|c|c|c|}
\hline Station & Code & Lat. $\left({ }^{\circ} \mathrm{N}\right)$ & Long. $\left({ }^{\circ} \mathrm{E}\right)$ & Elev. (m) & Climate & Data period & Samples & Mean GHI $\left(W / m^{2}\right)$ \\
\hline Alert & ALE & 82.490 & -62.420 & 127 & HA & 2009-2011 & 631284 & 223.84 \\
\hline Alice Springs & ASP & -23.798 & 133.888 & 547 & AR & 2007-2009 & 704784 & 561.96 \\
\hline Bermuda & BER & 32.267 & -64.667 & 8 & TM & 2006-2008 & 458837 & 497.33 \\
\hline Billings & BIL & 36.605 & -97.516 & 317 & TM & 2005-2007 & 631822 & 451.65 \\
\hline Bondville & BON & 40.067 & -88.367 & 213 & $\mathrm{TM}$ & 2007-2009 & 220046 & 507.54 \\
\hline Boulder & BOU & 40.050 & -105.007 & 1577 & TM & 2002-2004 & 481571 & 519.33 \\
\hline Brasilia & BRB & -15.601 & -47.713 & 1023 & TR & 2009-2011 & 598116 & 472.22 \\
\hline Carpentras & CAR & 44.083 & 5.059 & 100 & TM & 2003-2005 & 619585 & 419.19 \\
\hline Chesapeake Light & CLH & 36.905 & -75.713 & 37 & TM & 2011-2013 & 681269 & 408.60 \\
\hline Cener & CNR & 42.816 & -1.601 & 471 & TM & 2010-2012 & 666202 & 374.60 \\
\hline Cocos Island & $\mathrm{COC}$ & -12.193 & 96.835 & 6 & TR & 2006-2008 & 556163 & 505.99 \\
\hline De Aar & DAA & -30.667 & 23.993 & 1287 & $\mathrm{AR}$ & 2002-2004 & 592787 & 518.07 \\
\hline Darwin & DAR & -12.425 & 130.891 & 30 & TR & 2009-2011 & 699416 & 489.94 \\
\hline Concordia station & DOM & -75.100 & 123.383 & 3233 & HA & 2005-2007 & 255370 & 377.86 \\
\hline Desert Rock & DRA & 36.626 & -116.018 & 1007 & AR & 2007-2009 & 324644 & 596.47 \\
\hline Évora & EVR & 38.568 & -7.912 & 293 & TM & 2016-2017 & 199169 & 504.11 \\
\hline Eureka & EUR & 79.989 & -85.940 & 85 & HA & 2009-2011 & 654421 & 230.99 \\
\hline Fort Peck & FPE & 48.317 & -105.100 & 634 & TM & 2007-2009 & 227621 & 481.02 \\
\hline Fukuoka & FUA & 33.582 & 130.376 & 3 & TM & 2011-2013 & 715368 & 337.10 \\
\hline Goodwin Creek & GCR & 34.250 & -89.870 & 98 & TM & 2007-2009 & 244471 & 529.24 \\
\hline Gobabeb & GOB & -23.561 & 15.042 & 407 & AR & 2012-2014 & 627165 & 596.64 \\
\hline Georg von Neumayer & GVN & -70.650 & -8.250 & 42 & HA & 2011-2013 & 509631 & 316.63 \\
\hline Ilorin & ILO & 8.533 & 4.567 & 350 & TR & $1995,1999,2000$ & 160661 & 307.77 \\
\hline Ishigakijima & ISH & 24.337 & 124.163 & 6 & TM & 2011-2013 & 710421 & 374.30 \\
\hline Izana & IZA & 28.309 & -16.499 & 2373 & $\mathrm{AR}$ & $2011-2013$ & 680660 & 612.14 \\
\hline Kwajalein & KWA & 8.720 & 167.731 & 10 & $\mathrm{TR}$ & $1998-2000$ & 517467 & 544.96 \\
\hline Lauder & LAU & -45.045 & 169.689 & 350 & TM & 2005-2007 & 583349 & 399.17 \\
\hline Lerwick & LER & 60.139 & -1.185 & 80 & TM & 2004-2006 & 586958 & 213.18 \\
\hline Lindenberg & LIN & 52.210 & 14.122 & 125 & TM & $2001-2003$ & 665675 & 285.77 \\
\hline Momote & MAN & -2.058 & 147.425 & 6 & TR & $2008-2010$ & 689159 & 470.82 \\
\hline Minamitorishima & MNM & 24.288 & 153.983 & 7 & TM & $2011-2013$ & 727974 & 470.67 \\
\hline Nauru Island & NAU & -0.521 & 166.917 & 7 & TR & $2005-2007$ & 649304 & 513.96 \\
\hline Ny-Alesund & NYA & 78.925 & 11.930 & 11 & HA & $2007-2009$ & 619576 & 187.06 \\
\hline Palaiseau & PAL & 48.713 & 2.208 & 156 & TM & 2009-2011 & 701389 & 302.24 \\
\hline Payerne & PAY & 46.815 & 6.944 & 491 & TM & $2008-2010$ & 573646 & 349.50 \\
\hline Rock Springs & PSU & 40.720 & -77.933 & 376 & $\mathrm{TM}$ & 2007-2009 & 203868 & 471.41 \\
\hline Petrolina & PTR & -9.068 & -40.319 & 387 & TR & 2007-2009 & 149097 & 566.04 \\
\hline Regina & REG & 50.205 & -104.713 & 578 & TM & 2009-2011 & 620001 & 365.50 \\
\hline Sapporo & SAP & 43.060 & 141.328 & 17 & $\mathrm{AR}$ & 2011-2013 & 722699 & 305.91 \\
\hline Sede Boqer & SBO & 30.860 & 34.779 & 480 & TM & 2009-2011 & 662564 & 542.45 \\
\hline Sonnblick & SON & 47.054 & 12.958 & 3109 & HA & 2013-2015 & 462080 & 371.23 \\
\hline Solar Village & SOV & 24.907 & 46.397 & 768 & $\mathrm{AR}$ & $2000-2002$ & 717011 & 564.91 \\
\hline Sioux Falls & SXF & 43.730 & -96.620 & 473 & TM & 2007-2009 & 228600 & 475.41 \\
\hline Tamanrasset & TAM & 22.790 & 5.529 & 1385 & $\mathrm{AR}$ & $2006-2008$ & 644908 & 596.17 \\
\hline Tateno & TAT & 36.050 & 140.133 & 25 & TM & $2008-2010$ & 683402 & 334.39 \\
\hline Tiksi & TIK & 71.586 & 128.919 & 48 & HA & 2011-2013 & 617716 & 211.72 \\
\hline Toravere & TOR & 58.254 & 26.462 & 70 & TM & 2010-2012 & 649189 & 245.32 \\
\hline Xianghe & XIA & 39.754 & 116.962 & 32 & TM & $2008-2010$ & 561393 & 381.54 \\
\hline
\end{tabular}

for the fitting of the model parameters $A$ and $B$, since measurements of diffuse irradiance higher than global irradiance are very dubious. However, instrumental errors can occur, and therefore, the $K_{d}$ maximum value was set to 1.2 when determining the parameter $n$. Finally, measurements taken when the zenith angle was higher than $85^{\circ}$ were also removed due to disturbances caused mainly by the horizon line and also due to instrumental and modelling accuracy issues in that case [14]. Since 1-min data was used in this work, the extraterrestrial irradiance on a horizontal surface, $E_{o h}$, that is needed to determine the $K_{t}$ was simply calculated based on the solar constant $\left(G_{s}=1361.1 \mathrm{Wm}^{-2}\right.$ [77]) and the solar zenith angle, this last being calculated through the very accurate solar position algorithm developed by Reda and Andreas [78]. The data was divided into two sets: the training set with two years of data used to determine the fitting parameters of the proposed model; and the validation set with one year of data, used to validate the developed model as well as the models available in the literature (Section 2). These datasets are composed of years with high number of valid measurements, i.e., records that successfully passed the quality control procedures [76], as shown in Table 2.

\subsection{Statistical indicators for model assessment}

The developed model, as well as the models reviewed in Section 2, were evaluated using the statistical indicators described below taking the measured values as reference. Lower values indicate better model accuracy except for the mean bias error and mean percentage error, in which values closer to zero indicate a better model accuracy, and for the correlation coefficient, in which a value closer to 1 represents better model accuracy. In the following, $H$ and $N$ stand for minutely diffuse horizontal irradiance (DHI) and number of observations, respectively, and the subscripts $m, e$ and avg stand for measured, estimated and average, respectively.

\subsubsection{Mean bias error (MBE)}

$\mathrm{MBE}=\frac{1}{N} \sum_{i=1}^{N}\left(H_{e, i}-H_{m, i}\right)$ 

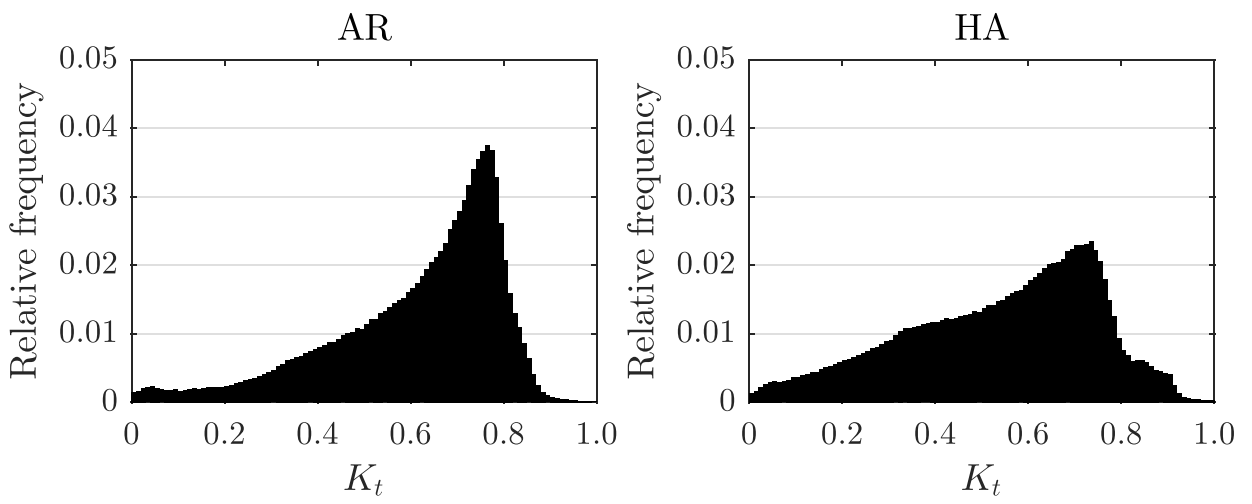

Fig. 3. Relative frequency of $K_{t}$ in BSRN stations according to the climate zone.

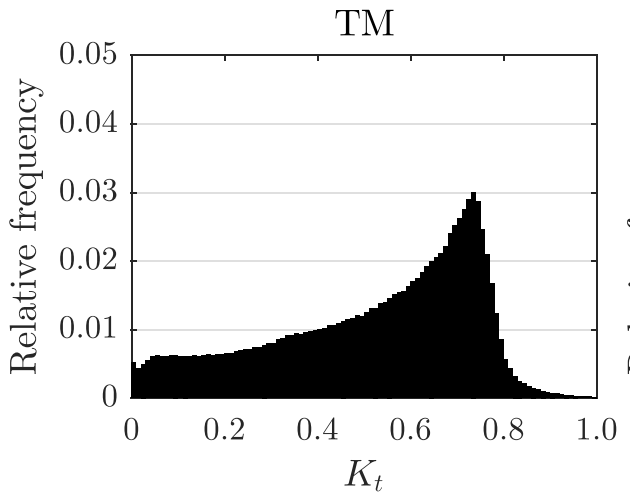

3.3.2. Mean absolute error (MAE)

$\operatorname{MAE}=\frac{1}{N} \sum_{i=1}^{N}\left|H_{e, i}-H_{m, i}\right|$

\subsubsection{Root mean square error (RMSE)}

$\mathrm{RMSE}=\left[\frac{1}{N} \sum_{i=1}^{N}\left(H_{e, i}-H_{m, i}\right)^{2}\right]^{\frac{1}{2}}$

(6)

3.3.4. Mean percentage error (MPE)

MPE $=\frac{1}{N} \sum_{i=1}^{N}\left(\frac{H_{e, i}-H_{m, i}}{H_{m, i}}\right) \times 100$

\subsubsection{Uncertainty at 95\% (U95)}

$\mathrm{U} 95=1.96\left(S D^{2}+R M S E^{2}\right)^{\frac{1}{2}}$

where $S D$ represents the standard deviation of the difference between $H_{e}$ and $H_{m}$.

\subsubsection{Relative root mean square error (RRMSE)}

RRMSE $=\frac{R M S E}{H_{m, a v g}}$

\subsubsection{T-statistics (TSTAT)}

$\operatorname{TSTAT}=\left[\frac{(N-1) M B E^{2}}{R M S E^{2}-M B E^{2}}\right]^{\frac{1}{2}}$
3.3.8. Maximum absolute relative error (erMAX)

$\operatorname{erMAX}=\max \left\{\left|\frac{H_{e, i}-H_{m, i}}{H_{m, i}}\right|, i=1, \ldots, N\right\}$

\subsubsection{Correlation coefficient (R)}

$\mathrm{R}=\frac{\sum_{i=1}^{N}\left(H_{e, i}-H_{e, \text { avg }}\right)\left(H_{m, i}-H_{m, \text { avg }}\right)}{\sqrt{\sum_{i=1}^{N}\left(H_{e, i}-H_{e, \text { avg }}\right)^{2} \sum_{i=1}^{N}\left(H_{m, i}-H_{m, \text { avg }}\right)^{2}}}$

\subsubsection{Mean absolute relative error (MARE)}

$\operatorname{MARE}=\frac{1}{N} \sum_{i=1}^{N}\left|\frac{H_{e, i}-H_{m, i}}{H_{m, i}}\right|$

\subsubsection{Global performance index (GPI)}

The global performance index, firstly proposed by Behar et al. [79], then modified by Despotovic et al. [80] and used by Jamil and Akthar $[23,81]$, is also used here to combine all the statistical indicators presented in Subsections 3.3.1 to 3.3.10. The need for using this index is due to the incapacity of those statistical indicators to, consistently, identify the best model (see Table 4). The values of the statistical indicators need to be scaled between 0 (worst performing model) and 1 (best performing model) to determine the GPI values, which otherwise would make difficult to compare the models. This normalisation also allows using the same statistical weight for all of the indicators when determining the GPI, as follows:

$\mathrm{GPI}=\sum_{j=1}^{10} \alpha_{j}\left(\bar{y}_{j}-y_{i j}\right)$

where $\bar{y}_{j}$ is the median of the scaled values of the indicator $j, y_{i j}$ is the scaled value of the statistical indicator $j$ for the model $i$, and $\alpha_{j}$ equals to 1 for all statistical indicators except $R$, in which $\alpha_{j}$ equals to -1 . The GPI also allows to combine several indicators regardless if the value of a single indicator is 0 or not, which is not possible if a simple product of 
the indicators is computed, and therefore a higher GPI stands for better accuracy of a given model.

\section{Results and discussion}

\subsection{Determination of model parameters and climate analysis}

Fig. 3 shows the distribution of the $K_{t}$ values for the climate zones considered, which is useful to identify the range of the clearness index and the clear sky occurrences (frequency). The AR climate zone presents the highest relative frequency for high values of $K_{t}$, reaching a relative frequency of 0.038 for $K_{t} \simeq 0.77$, followed by the TM and TR climate zones with maximum relative frequency around 0.030 for approximately the same $K_{t}$ value. The HA climate zone presents the lower values of $K_{t}$ relative frequency for clear sky.

The training of the model was performed using the stations from the BSRN network, while the IES station (EVR) was used only in the validation of the model. The parameters $A$ and $B$ for each station were found by fitting Eq. (2) to the data in the range of $K_{t} \geq 0.5$, using the non-linear least squares method. Then, the parameter $n$ was obtained through an optimisation process in order to achieve the maximum GPI value for the entire range of the data. One also investigated the existence of a possible relationship between these parameters and the elevation of the stations according to the climate zone, as shown in Fig. 4. However no conclusions can be drawn on the existence of any clear dependence, and therefore, no traditional fitting equations using the parameters of the model and the elevation of the stations were able to present an acceptable coefficient of determination.

To develop a model based only on the $K_{t}$ as predictor for each climate region, the mean value and standard deviation of these parameters were calculated for the four zones considered, and the stations in which at least one of the three parameters were out of the confidence interval defined by the mean \pm standard deviation were excluded from

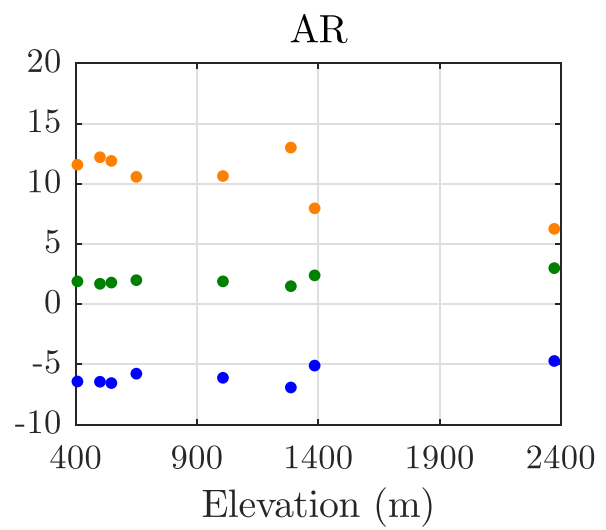

TM

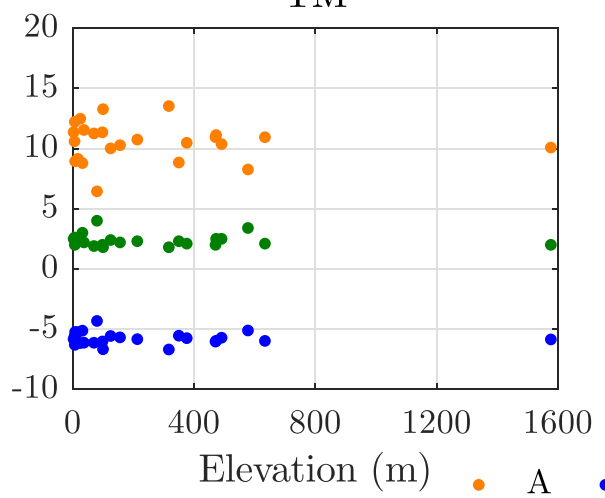

Table 3

Parameters of the developed model according to the climate zone: Arid (AR), High Albedo (HA), Temperate (TM) and Tropical (TR).

\begin{tabular}{lllll}
\hline Parameters & \multicolumn{4}{l}{ Climate Zone } \\
\hline & AR & HA & TM & TR \\
A & 11.39 & 7.83 & 10.79 & 11.59 \\
B & -6.25 & -4.59 & -5.87 & -6.14 \\
$\mathrm{n}$ & 1.86 & 3.25 & 2.24 & 1.87 \\
\hline
\end{tabular}

this calculation. After this procedure, the values inside this imposed range are averaged in order to obtain the mean values of $A, B$ and $n$ for each climate zone, as shown in Table 3 . These parameters were used for the model performance assessment as presented in the following section.

\subsection{Performance assessment}

The statistical tools presented in Section 3.3 were used to assess the performance of the models from the literature as well as of the model developed in this work using measurements from the EVR station and the datasets from the BSRN, as presented in Table 2. The performance assessment was carried out using the corresponding set of parameters according to the climate zone of the 48 radiometric stations analysed in this study. The performance assessment is presented in detail for the EVR station as an example of both the methodology used in this study and as a completely independent assessment since data from this station was not included in the determination of the model parameters. Table 4 presents the results of the statistical analysis of the selected models using the indicators shown in Section 3.3 for the EVR station. The bold font indicates the optimal values of the statistical indicators, i.e., it indicates the best model according to each statistical indicator.

The statistical evaluation regarding the accuracy of the models
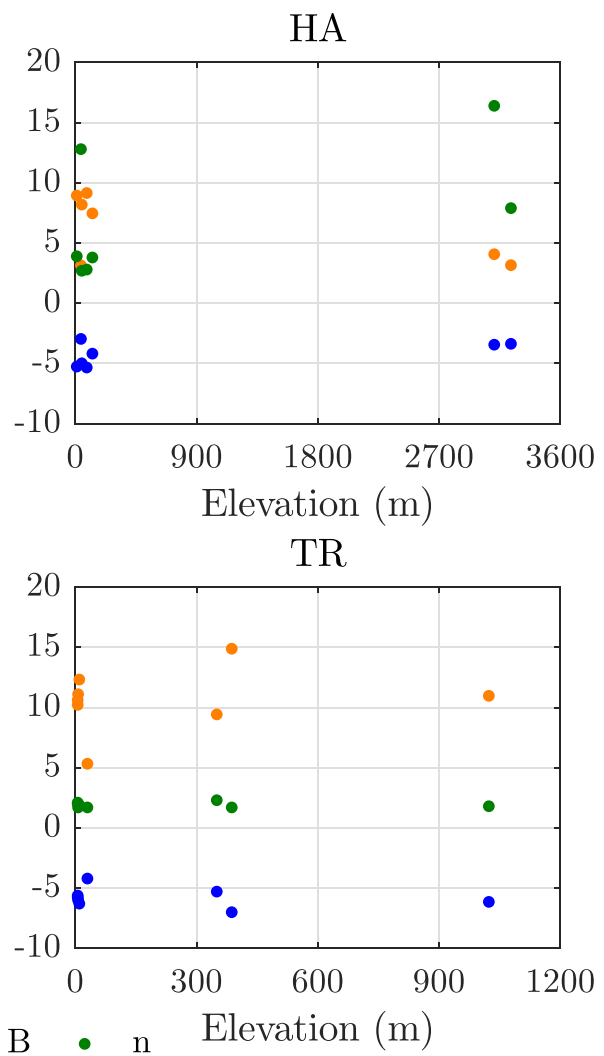

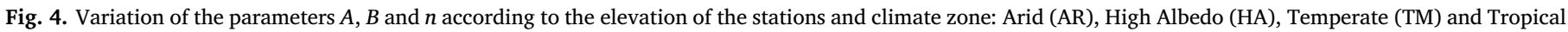
(TR). 
Table 4

Statistical analysis of the selected models for the EVR station.

\begin{tabular}{|c|c|c|c|c|c|c|c|c|c|c|c|}
\hline Model & $\operatorname{MBE}\left(W m^{-2}\right)$ & RMSE $\left(W m^{-2}\right)$ & TSTAT & MPE (\%) & U95 $\left(W m^{-2}\right)$ & erMAX & RRMSE & $\operatorname{MAE}\left(W m^{-2}\right)$ & $\mathrm{R}$ & MARE & GPI \\
\hline 1 & -40.31 & 111.13 & 36.42 & -6.42 & 297.74 & 2.00 & 0.86 & 75.41 & 0.19 & 0.55 & 0.22 \\
\hline 2 & -29.87 & 102.79 & 28.42 & 3.82 & 278.84 & 2.11 & 0.79 & 71.26 & 0.30 & 0.54 & 0.64 \\
\hline 3 & -46.36 & 112.67 & 42.25 & -12.76 & 298.80 & 1.79 & 0.87 & 75.05 & 0.19 & 0.53 & 0.13 \\
\hline 4 & -74.08 & 124.44 & 69.34 & -41.84 & 312.87 & 0.89 & 0.96 & 79.87 & 0.19 & 0.50 & -0.46 \\
\hline 5 & -79.12 & 127.30 & 74.25 & -47.13 & 316.96 & 0.90 & 0.98 & 82.42 & 0.19 & 0.52 & -0.63 \\
\hline 6 & -74.08 & 124.44 & 69.34 & -41.84 & 312.87 & 0.89 & 0.96 & 79.87 & 0.19 & 0.50 & -0.46 \\
\hline 7 & -99.29 & 140.53 & 93.43 & -68.28 & 337.45 & 0.94 & 1.08 & 99.29 & 0.19 & 0.68 & -1.46 \\
\hline 8 & -79.12 & 127.30 & 74.25 & -47.13 & 316.96 & 0.90 & 0.98 & 82.42 & 0.19 & 0.52 & -0.63 \\
\hline 9 & -53.92 & 115.14 & 49.60 & -20.69 & 301.16 & 1.54 & 0.89 & 75.16 & 0.19 & 0.51 & 0.00 \\
\hline 10 & -79.12 & 127.30 & 74.25 & -47.13 & 316.96 & 0.90 & 0.98 & 82.42 & 0.19 & 0.52 & -0.63 \\
\hline 11 & -69.04 & 121.78 & 64.41 & -36.56 & 309.23 & 1.03 & 0.94 & 77.95 & 0.19 & 0.49 & -0.32 \\
\hline 12 & -58.96 & 117.11 & 54.53 & -25.98 & 303.36 & 1.37 & 0.90 & 75.67 & 0.19 & 0.50 & -0.10 \\
\hline 13 & -94.25 & 136.99 & 88.73 & -62.99 & 331.75 & 0.93 & 1.06 & 94.31 & 0.19 & 0.63 & -1.24 \\
\hline 14 & -79.12 & 127.30 & 74.25 & -47.13 & 316.96 & 0.90 & 0.98 & 82.42 & 0.19 & 0.52 & -0.63 \\
\hline 15 & -89.21 & 133.59 & 83.95 & -57.70 & 326.42 & 0.92 & 1.03 & 89.69 & 0.19 & 0.58 & -1.02 \\
\hline 16 & -79.12 & 127.30 & 74.25 & -47.13 & 316.96 & 0.90 & 0.98 & 82.42 & 0.19 & 0.52 & -0.63 \\
\hline 17 & -21.15 & 109.03 & 18.51 & 13.67 & 299.35 & 2.64 & 0.84 & 78.71 & 0.19 & 0.64 & 0.24 \\
\hline 18 & 28.34 & 79.71 & 35.61 & 56.30 & 213.84 & 2.98 & 0.62 & 63.06 & 0.68 & 0.69 & 0.91 \\
\hline 19 & -48.88 & 113.43 & 44.69 & -15.41 & 299.45 & 1.71 & 0.88 & 75.01 & 0.19 & 0.52 & 0.09 \\
\hline 20 & -38.25 & 107.47 & 35.65 & -3.12 & 288.30 & 2.10 & 0.83 & 69.93 & 0.24 & 0.49 & 0.45 \\
\hline 21 & 1.53 & 112.10 & 1.28 & 37.46 & 310.72 & 3.40 & 0.87 & 85.97 & 0.19 & 0.78 & -0.01 \\
\hline 22 & -16.11 & 109.19 & 13.96 & 18.96 & 301.01 & 2.81 & 0.84 & 80.05 & 0.19 & 0.67 & 0.20 \\
\hline 23 & 0.42 & 82.32 & 0.48 & 28.96 & 228.18 & 2.45 & 0.64 & 54.46 & 0.59 & 0.51 & 1.66 \\
\hline 24 & -55.43 & 115.71 & 51.08 & -22.28 & 301.76 & 1.49 & 0.89 & 75.28 & 0.19 & 0.50 & -0.03 \\
\hline 25 & -53.41 & 114.96 & 49.11 & -20.17 & 300.96 & 1.56 & 0.89 & 75.13 & 0.19 & 0.51 & 0.01 \\
\hline 26 & -40.31 & 111.13 & 36.42 & -6.42 & 297.74 & 2.00 & 0.86 & 75.41 & 0.19 & 0.55 & 0.22 \\
\hline 27 & -30.22 & 109.49 & 26.88 & 4.15 & 297.65 & 2.34 & 0.85 & 76.76 & 0.19 & 0.59 & 0.29 \\
\hline 28 & 3.55 & 112.66 & 2.95 & 39.58 & 312.20 & 3.47 & 0.87 & 86.76 & 0.19 & 0.80 & -0.09 \\
\hline 29 & 19.18 & 118.40 & 15.36 & 55.97 & 326.03 & 3.99 & 0.91 & 93.67 & 0.19 & 0.91 & -0.69 \\
\hline 30 & 8.09 & 114.07 & 6.65 & 44.34 & 315.79 & 3.62 & 0.88 & 88.64 & 0.19 & 0.83 & -0.26 \\
\hline 31 & -99.29 & 140.53 & 93.43 & -68.28 & 337.45 & 0.94 & 1.08 & 99.29 & 0.19 & 0.68 & -1.46 \\
\hline 32 & -56.45 & 105.00 & 59.68 & -26.47 & 269.19 & 1.77 & 0.81 & 60.44 & 0.49 & 0.33 & 0.64 \\
\hline 33 & -47.02 & 121.55 & 39.26 & -12.54 & 324.08 & 2.03 & 0.94 & 82.07 & 0.08 & 0.60 & -0.29 \\
\hline 34 & -129.12 & 164.09 & 119.34 & -99.48 & 377.92 & 1.00 & 1.27 & 129.12 & 0.18 & 0.99 & -2.82 \\
\hline 35 & 14.18 & 77.91 & 17.32 & 42.93 & 214.17 & 2.63 & 0.60 & 58.39 & 0.65 & 0.60 & 1.37 \\
\hline 36 & -38.79 & 110.81 & 34.98 & -4.83 & 297.59 & 2.05 & 0.86 & 75.56 & 0.19 & 0.56 & 0.24 \\
\hline 37 & -13.59 & 109.39 & 11.72 & 21.60 & 302.03 & 2.89 & 0.84 & 80.77 & 0.19 & 0.69 & 0.18 \\
\hline 38 & 21.99 & 87.63 & 24.26 & 45.07 & 239.04 & 5.94 & 0.68 & 60.85 & 0.61 & 0.60 & 0.49 \\
\hline 39 & 21.70 & 119.55 & 17.27 & 58.61 & 328.63 & 4.08 & 0.92 & 94.89 & 0.19 & 0.93 & -0.79 \\
\hline 40 & 21.70 & 119.55 & 17.27 & 58.61 & 328.63 & 4.08 & 0.92 & 94.89 & 0.19 & 0.93 & -0.79 \\
\hline 41 & -28.71 & 109.34 & 25.47 & 5.74 & 297.82 & 2.39 & 0.84 & 77.04 & 0.19 & 0.60 & 0.28 \\
\hline 42 & 91.34 & 162.12 & 63.82 & 130.84 & 412.18 & 6.25 & 1.25 & 131.34 & 0.23 & 1.48 & -3.61 \\
\hline 43 & -59.97 & 117.54 & 55.52 & -27.04 & 303.86 & 1.34 & 0.91 & 75.82 & 0.19 & 0.50 & -0.12 \\
\hline 44 & -38.79 & 110.81 & 34.98 & -4.83 & 297.59 & 2.05 & 0.86 & 75.56 & 0.19 & 0.56 & 0.24 \\
\hline 45 & 4.17 & 75.61 & 5.17 & 28.26 & 209.41 & 4.19 & 0.58 & 52.45 & 0.67 & 0.47 & 1.56 \\
\hline 46 & 35.49 & 81.81 & 45.07 & 63.51 & 215.83 & 3.10 & 0.63 & 65.77 & 0.69 & 0.74 & 0.64 \\
\hline 47 & -40.31 & 111.13 & 36.42 & -6.42 & 297.74 & 2.00 & 0.86 & 75.41 & 0.19 & 0.55 & 0.22 \\
\hline 48 & -20.14 & 109.03 & 17.59 & 14.73 & 299.64 & 2.67 & 0.84 & 78.97 & 0.19 & 0.65 & 0.23 \\
\hline 49 & -63.50 & 119.10 & 58.98 & -30.74 & 305.76 & 1.22 & 0.92 & 76.47 & 0.19 & 0.49 & -0.20 \\
\hline 50 & -22.16 & 109.03 & 19.43 & 12.61 & 299.08 & 2.61 & 0.84 & 78.47 & 0.19 & 0.64 & 0.25 \\
\hline 51 & 0.97 & 74.95 & 1.21 & 27.30 & 207.75 & 2.63 & 0.58 & 50.05 & 0.67 & 0.47 & 1.92 \\
\hline 52 & 80.04 & 123.80 & 79.32 & 114.80 & 305.19 & 4.66 & 0.96 & 101.68 & 0.51 & 1.22 & -1.94 \\
\hline 53 & -1.70 & 75.32 & 2.12 & 25.91 & 208.75 & 2.26 & 0.58 & 51.50 & 0.67 & 0.47 & 1.96 \\
\hline 54 & 25.21 & 82.06 & 30.22 & 54.78 & 222.02 & 2.88 & 0.63 & 64.16 & 0.64 & 0.69 & 0.89 \\
\hline 55 & 22.66 & 80.81 & 27.34 & 51.93 & 219.55 & 2.85 & 0.62 & 62.57 & 0.64 & 0.67 & 1.01 \\
\hline 56 & 8.51 & 75.99 & 10.54 & 36.20 & 209.99 & 2.64 & 0.59 & 54.50 & 0.67 & 0.54 & 1.62 \\
\hline 57 & -2.72 & 74.91 & 3.40 & 24.30 & 207.57 & 2.33 & 0.58 & 50.11 & 0.67 & 0.46 & 1.97 \\
\hline 58 & 17.72 & 78.78 & 21.61 & 46.49 & 215.59 & 2.77 & 0.61 & 59.71 & 0.65 & 0.62 & 1.23 \\
\hline 59 & 5.25 & 75.53 & 6.53 & 32.87 & 209.11 & 2.52 & 0.58 & 53.32 & 0.67 & 0.52 & 1.75 \\
\hline 60 & -48.27 & 108.42 & 46.53 & -19.98 & 285.25 & 2.48 & 0.84 & 57.67 & 0.42 & 0.36 & 0.56 \\
\hline 61 & 38.92 & 113.31 & 34.23 & 75.18 & 304.68 & 4.23 & 0.87 & 92.15 & 0.31 & 0.97 & -0.91 \\
\hline 62 & -106.85 & 146.12 & 100.33 & -76.21 & 346.67 & 0.96 & 1.13 & 106.85 & 0.19 & 0.76 & -1.80 \\
\hline 63 & -0.89 & 111.49 & 0.74 & 34.92 & 309.04 & 3.32 & 0.86 & 85.04 & 0.19 & 0.77 & 0.06 \\
\hline 64 & 32.23 & 124.95 & 24.99 & 69.66 & 340.53 & 4.43 & 0.96 & 100.26 & 0.19 & 1.01 & -1.23 \\
\hline 65 & -72.77 & 123.26 & 68.46 & -39.64 & 310.46 & 2.34 & 0.95 & 74.37 & 0.23 & 0.42 & -0.50 \\
\hline 66 & -57.44 & 104.79 & 61.34 & -28.93 & 267.76 & 2.56 & 0.81 & 65.17 & 0.51 & 0.39 & 0.43 \\
\hline 67 & 17.89 & 77.44 & 22.22 & 44.70 & 211.78 & 2.80 & 0.60 & 58.85 & 0.67 & 0.60 & 1.30 \\
\hline 68 & 17.38 & 74.36 & 22.50 & 44.68 & 203.28 & 2.59 & 0.57 & 57.34 & 0.70 & 0.60 & 1.44 \\
\hline 69 & 54.08 & 108.58 & 53.76 & 86.97 & 281.68 & 4.31 & 0.84 & 87.56 & 0.47 & 0.99 & -0.92 \\
\hline 70 & 10.66 & 75.44 & 13.35 & 38.81 & 208.07 & 6.90 & 0.58 & 56.61 & 0.68 & 0.56 & 0.86 \\
\hline 71 & 8.27 & 74.35 & 10.48 & 36.44 & 205.45 & 2.64 & 0.57 & 55.35 & 0.69 & 0.55 & 1.66 \\
\hline 72 & -24.73 & 80.84 & 30.08 & 2.11 & 218.76 & 1.88 & 0.62 & 47.74 & 0.66 & 0.33 & 1.87 \\
\hline 73 & -7.55 & 78.20 & 9.08 & 21.51 & 216.24 & 2.09 & 0.60 & 55.36 & 0.66 & 0.47 & 1.84 \\
\hline 74 & 18.79 & 80.38 & 22.50 & 48.26 & 219.75 & 2.67 & 0.62 & 61.71 & 0.64 & 0.64 & 1.15 \\
\hline
\end{tabular}


Table 4 (continued)

\begin{tabular}{|c|c|c|c|c|c|c|c|c|c|c|c|}
\hline Model & $\operatorname{MBE}\left(W m^{-2}\right)$ & RMSE $\left(W m^{-2}\right)$ & TSTAT & MPE (\%) & $\mathrm{U} 95\left(W m^{-2}\right)$ & erMAX & RRMSE & $\operatorname{MAE}\left(W m^{-2}\right)$ & $\mathrm{R}$ & MARE & GPI \\
\hline 75 & 3.70 & 76.96 & 4.50 & 32.28 & 213.19 & 2.17 & 0.59 & 55.20 & 0.65 & 0.52 & 1.78 \\
\hline 76 & -16.42 & 77.36 & 20.33 & 10.66 & 211.99 & 1.89 & 0.60 & 49.07 & 0.68 & 0.38 & 1.97 \\
\hline 77 & -20.39 & 80.17 & 24.61 & 6.91 & 218.59 & 1.91 & 0.62 & 48.59 & 0.65 & 0.36 & 1.87 \\
\hline 78 & -9.96 & 78.53 & 11.97 & 18.35 & 216.80 & 1.92 & 0.61 & 51.70 & 0.64 & 0.43 & 1.88 \\
\hline 79 & -32.59 & 109.77 & 29.10 & 1.67 & 297.48 & 2.26 & 0.85 & 76.37 & 0.19 & 0.58 & 0.29 \\
\hline 80 & -39.45 & 110.95 & 35.60 & -5.52 & 297.65 & 2.03 & 0.86 & 75.49 & 0.19 & 0.55 & 0.23 \\
\hline 81 & -41.06 & 111.30 & 37.15 & -7.21 & 297.83 & 1.97 & 0.86 & 75.35 & 0.19 & 0.55 & 0.21 \\
\hline 82 & -38.79 & 110.81 & 34.98 & -4.83 & 297.59 & 2.05 & 0.86 & 75.56 & 0.19 & 0.56 & 0.24 \\
\hline 83 & -27.20 & 109.23 & 24.06 & 7.33 & 298.03 & 2.44 & 0.84 & 77.34 & 0.19 & 0.61 & 0.27 \\
\hline 84 & -26.54 & 109.18 & 23.46 & 8.01 & 298.14 & 2.46 & 0.84 & 77.48 & 0.19 & 0.61 & 0.27 \\
\hline 85 & -8.55 & 110.00 & 7.29 & 26.89 & 304.45 & 3.06 & 0.85 & 82.35 & 0.19 & 0.72 & 0.14 \\
\hline 86 & -64.00 & 119.33 & 59.47 & -31.27 & 306.06 & 1.20 & 0.92 & 76.58 & 0.19 & 0.49 & -0.21 \\
\hline 87 & 84.34 & 128.86 & 81.01 & 116.82 & 316.63 & 5.10 & 0.99 & 103.80 & 0.50 & 1.24 & -2.17 \\
\hline 88 & 6.58 & 113.58 & 5.43 & 42.75 & 314.55 & 3.57 & 0.88 & 88.00 & 0.19 & 0.82 & -0.20 \\
\hline 89 & 92.63 & 132.94 & 90.92 & 126.87 & 320.66 & 5.13 & 1.03 & 108.04 & 0.51 & 1.32 & -2.49 \\
\hline 90 & 88.67 & 123.26 & 96.91 & 119.65 & 294.17 & 4.67 & 0.95 & 100.93 & 0.61 & 1.24 & -2.00 \\
\hline 91 & 87.44 & 121.89 & 96.37 & 119.29 & 291.17 & 4.63 & 0.94 & 100.66 & 0.61 & 1.24 & -1.97 \\
\hline 92 & -179.35 & 287.33 & 74.77 & -141.81 & 714.66 & 5.68 & 2.22 & 202.48 & -0.30 & 1.59 & -6.35 \\
\hline 93 & -18.41 & 72.93 & 24.41 & 5.27 & 198.91 & 2.15 & 0.56 & 44.95 & 0.72 & 0.36 & 2.08 \\
\hline 94 & -11.85 & 75.52 & 14.87 & 14.47 & 208.03 & 2.23 & 0.58 & 47.25 & 0.68 & 0.39 & 1.98 \\
\hline 95 & -5.82 & 71.58 & 7.64 & 19.72 & 198.07 & 2.30 & 0.55 & 47.76 & 0.72 & 0.41 & 2.12 \\
\hline 96 & -60.98 & 121.20 & 54.48 & -33.86 & 313.97 & 2.74 & 0.94 & 67.90 & 0.38 & 0.46 & -0.10 \\
\hline 97 & -79.12 & 127.30 & 74.25 & -47.13 & 316.96 & 0.90 & 0.98 & 82.42 & 0.19 & 0.52 & -0.63 \\
\hline 98 & -84.16 & 130.36 & 79.13 & -52.42 & 321.48 & 0.91 & 1.01 & 85.68 & 0.19 & 0.55 & -0.82 \\
\hline 99 & -74.08 & 124.44 & 69.34 & -41.84 & 312.87 & 0.89 & 0.96 & 79.87 & 0.19 & 0.50 & -0.46 \\
\hline 100 & -74.08 & 124.44 & 69.34 & -41.84 & 312.87 & 0.89 & 0.96 & 79.87 & 0.19 & 0.50 & -0.46 \\
\hline 101 & -69.04 & 121.78 & 64.41 & -36.56 & 309.23 & 1.03 & 0.94 & 77.95 & 0.19 & 0.49 & -0.32 \\
\hline 102 & -79.12 & 127.30 & 74.25 & -47.13 & 316.96 & 0.90 & 0.98 & 82.42 & 0.19 & 0.52 & -0.63 \\
\hline 103 & -69.04 & 121.78 & 64.41 & -36.56 & 309.23 & 1.03 & 0.94 & 77.95 & 0.19 & 0.49 & -0.32 \\
\hline 104 & -79.12 & 127.30 & 74.25 & -47.13 & 316.96 & 0.90 & 0.98 & 82.42 & 0.19 & 0.52 & -0.63 \\
\hline 105 & -58.96 & 117.11 & 54.53 & -25.98 & 303.36 & 1.37 & 0.90 & 75.67 & 0.19 & 0.50 & -0.10 \\
\hline 106 & -74.08 & 124.44 & 69.34 & -41.84 & 312.87 & 0.89 & 0.96 & 79.87 & 0.19 & 0.50 & -0.46 \\
\hline 107 & -58.96 & 117.11 & 54.53 & -25.98 & 303.36 & 1.37 & 0.90 & 75.67 & 0.19 & 0.50 & -0.10 \\
\hline 108 & -69.04 & 121.78 & 64.41 & -36.56 & 309.23 & 1.03 & 0.94 & 77.95 & 0.19 & 0.49 & -0.32 \\
\hline 109 & -64.00 & 119.33 & 59.47 & -31.27 & 306.06 & 1.20 & 0.92 & 76.58 & 0.19 & 0.49 & -0.21 \\
\hline 110 & -84.16 & 130.36 & 79.13 & -52.42 & 321.48 & 0.91 & 1.01 & 85.68 & 0.19 & 0.55 & -0.82 \\
\hline 111 & -69.04 & 121.78 & 64.41 & -36.56 & 309.23 & 1.03 & 0.94 & 77.95 & 0.19 & 0.49 & -0.32 \\
\hline 112 & -64.00 & 119.33 & 59.47 & -31.27 & 306.06 & 1.20 & 0.92 & 76.58 & 0.19 & 0.49 & -0.21 \\
\hline 113 & -64.00 & 119.33 & 59.47 & -31.27 & 306.06 & 1.20 & 0.92 & 76.58 & 0.19 & 0.49 & -0.21 \\
\hline 114 & -74.08 & 124.44 & 69.34 & -41.84 & 312.87 & 0.89 & 0.96 & 79.87 & 0.19 & 0.50 & -0.46 \\
\hline 115 & -15.14 & 76.83 & 18.81 & 11.50 & 210.89 & 2.11 & 0.59 & 47.82 & 0.67 & 0.38 & 1.95 \\
\hline 116 & -24.37 & 80.74 & 29.63 & 1.31 & 218.65 & 2.19 & 0.62 & 45.81 & 0.65 & 0.32 & 1.84 \\
\hline 117 & -6.69 & 76.75 & 8.19 & 20.13 & 212.34 & 2.34 & 0.59 & 49.23 & 0.66 & 0.43 & 1.91 \\
\hline 118 & -14.95 & 75.02 & 19.03 & 10.74 & 205.86 & 2.27 & 0.58 & 46.33 & 0.69 & 0.36 & 2.01 \\
\hline 119 & -2.52 & 74.47 & 3.16 & 23.97 & 206.36 & 2.44 & 0.57 & 49.39 & 0.68 & 0.45 & 1.98 \\
\hline 120 & -16.08 & 77.31 & 19.90 & 10.01 & 211.97 & 2.25 & 0.60 & 46.70 & 0.67 & 0.36 & 1.93 \\
\hline 121 & 1.42 & 92.87 & 1.44 & 34.45 & 257.41 & 2.85 & 0.72 & 69.35 & 0.42 & 0.62 & 0.95 \\
\hline This work & 25.84 & 70.80 & 36.68 & 47.87 & 189.61 & 2.59 & 0.55 & 53.70 & 0.76 & 0.58 & 1.43 \\
\hline
\end{tabular}

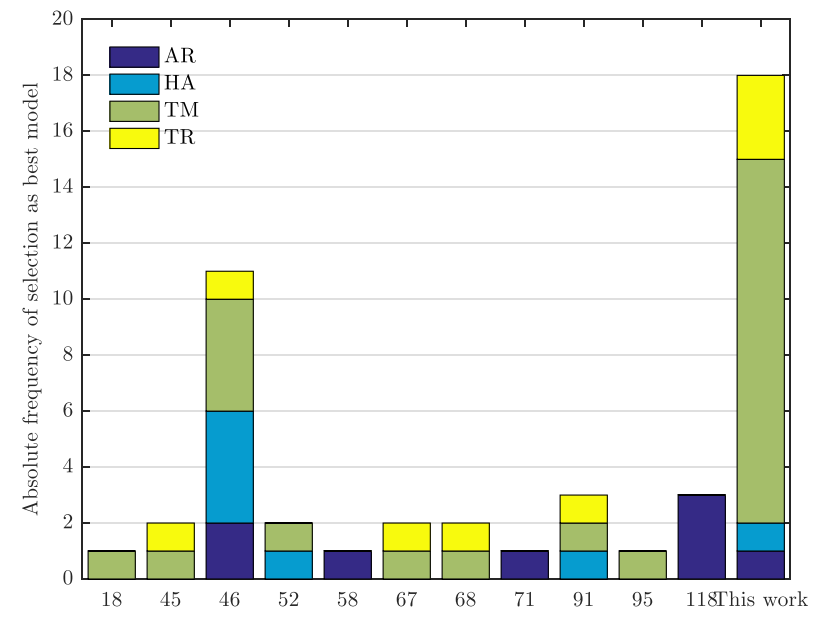

Fig. 5. Best performing models according to the climate zone: arid (AR), high albedo (HA), temperate (TM) and tropical (TR). presented in Table 4 for EVR does not give a unanimous decision on which of the models is considered the most accurate. The model developed in this work is the best performing model if we consider the RMSE (70.80 $\left.\mathrm{W} \mathrm{m}^{-2}\right)$, U95 (189.61 $\mathrm{W} \mathrm{m}^{-2}$ ), RRMSE (0.55) and R (0.76) statistical indicators. On the other hand, the model 23 presented by Hollands [31], is the most accurate model regarding the MBE $(0.42$ $W \mathrm{~m}^{-2}$ ) and TSTAT (0.48) indicators. Considering the values of MPE (1.31\%) and MARE (0.32) of the model 116 derived by Abal et al. [67], it would be selected as the best performing model for this station. Regarding the erMAX, it does not allow identifying the single most accurate model, due to presenting the same value for several models. The analysis of these results shows the advantage of using the GPI in order to present a more concise performance evaluation, allowing the combination of several statistical indicators and providing, through a simple procedure, a result easy to understand. Therefore, the model with higher GPI for the station being analysed (EVR) is the model 95 presented by Marques Filho et al. [65] with a GPI value of 2.12, although it was not considered the best performing model according to any of the statistical indicators separately. This result is due to the scaling down of the values of the statistical indicators mentioned above in the GPI determination procedure, which allows for a fair comparison of the 


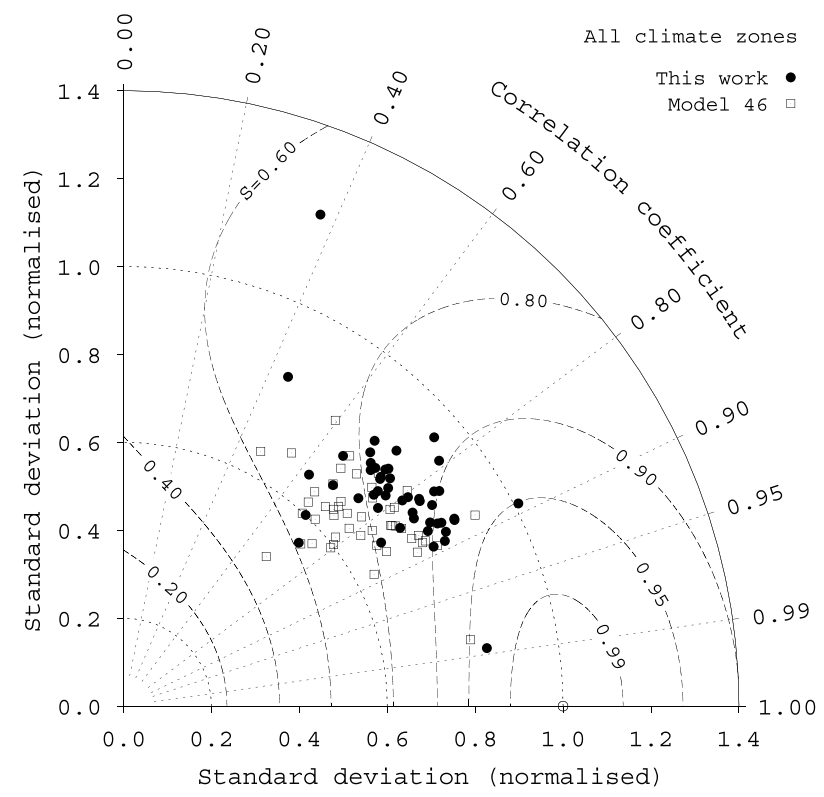

Fig. 6. Taylor diagram for the results of model 46 and the model developed in this work for all climate zones.

models under study.

This analysis was also carried out for all of the BSRN stations in order to identify the best performing model for various locations in different climate zones. Fig. 5 presents the result of this comprehensive performance assessment, where the absolute frequency of selection as the best performing model (based on the maximum GPI) is shown, also according to the climate zone of the stations.

The best overall performing model is the one presented in this work (Eq. (3) and Table 3), followed by model 46 by Mondol et al. [47]. The best performing model for the AR climate zone is the model 118 proposed by Abal et al. [67] while the best performing model for the HA climate zone is the model 46 derived by Mondol et al. [47]. The best performing model for the TM and TR climate zones is the model presented in this work (Eq. (3) and Table 3). The results presented in Fig. 5 show the advantage in deriving specific model parameters for each climate zone as suggested by Gueymard and Ruiz-Arias [14], despite the good performance of the model proposed by Mondol [47]. Only the model proposed here, and the model proposed by Mondol [47] were selected as best performing models for the four climate zones considered, showing excellent versatility regarding climate zone selection. On the other hand, the higher number of stations in the temperate (TM) zone allows for a better characterisation of the model parameters in comparison against other climate zones, where less data is available.

To further test the closeness of the two best performing models from Fig. 5 to the measured data, i.e., model 46 and the one proposed in this work, a Taylor diagram [25] is presented in Fig. 6 for all stations. This diagram helps to identify the closeness between the modelled and observed data in terms of their correlation coefficient (azimuthal position, $\mathrm{R}$ ) and standard deviation of model output normalised by the standard deviation of the corresponding observations (radial distance, nSD). In this diagram, a better accuracy is achieved when the model is located close to the normalised standard deviation unit line [14], while the open circle in the coordinates ( $\mathrm{nSD}=1, \mathrm{R}=1$ ) represents the point of perfect match between model and observations. It is worth to mention that the distance between the model representation and this point is the so-called centred pattern Root Mean Square Difference, which accounts for the mean values of both data series, and is also a measure of the model closeness to the observed data (not shown). Instead, a more elaborated skill score proposed by Taylor [25] was determined as a function of the correlation coefficient (R) and the normalised standard deviation (nSD) in the form $S=4(1+R) /\left[(n S D+1 / n S D)^{2}\left(1+R_{0}\right)\right]$ with $R_{0}=\max (R)$, which is represented in Fig. 6 by the $S$ isolines. This definition allows guaranteeing that the skillfulness of the model increases when $n S D \rightarrow 1$, which is not assured by the centred pattern RMSE for lower values of R. Best model accuracy is achieved when skill score values tend to unity. In this way, the best performing model according to Fig. 6 is the model proposed in this work, with a skill score higher than 0.80 for the majority of the stations and with nSD values in general higher than those of model 46. This conclusion is in agreement with the results presented in Fig. 5, showing that the proposed model has best accuracy, using two distinct statistical analysis.

\section{Conclusions}

In this work is presented a newly developed model based on minute resolution data and climate zone classification. A review of the literature on models that use hourly and sub-hourly $K_{t}$ values to compute $K_{d}$ was carried out. An extensive statistical analysis of the proposed as well as of other 121 models found in the literature was performed, using 1min resolution data. In this analysis, the elevation of the stations was not an essential factor when determining the parameters of the new model. The performance assessment was done using several statistical indicators and a Global Performance Index (GPI), which is a composite indicator that simplifies the analysis. The best performing model for the arid (AR) climate zone was found to be the model proposed by Abal et al. [67], while the best model for the high albedo (HA) climate zone is the model proposed by Mondol et al. [47]. The best model for the temperate (TM) and tropical (TR) climate zones is the model proposed in this work. It was also found that the best overall performing model (highest GPI in the more significant number of stations and highest skill score) was the model proposed in this work followed by the model by Mondol et al. [47]. This work helps to identify the best model that uses only the $K_{t}$ according to the climate zone, thus allowing to easily estimate the diffuse horizontal irradiance (DHI) and consequently the direct normal irradiance (DNI), based only on global horizontal irradiance (GHI) measurements. The proposed model is also a tool for longterm data series quality control and gap-filling.

\section{Acknowledgements}

Edgar F.M. Abreu acknowledges the support of the FCT (the Portuguese Science and Technology Foundation) through the grant with reference SFRH/BD/136433/2018. The authors acknowledge the funding provided by the European Union through the European Regional Development Fund, included in the COMPETE 2020 (Operational Program Competitiveness and Internationalization) through the ICT project (UID/GEO/04683/2013) with the reference POCI-01-0145-FEDER-007690 and the project DNI-Alentejo with reference ALT20-03-0145-FEDER-000011. The authors also acknowledge to the personnel who maintain the Baseline Surface Radiation Network radiometric stations.

\section{References}

[1] Engerer NA. Minute resolution estimates of the diffuse fraction of global irradiance for southeastern Australia. Sol Energy 2015;116:215-37. https://doi.org/10.1016/ j.solener.2015.04.012.

[2] Kaddoura TO, Ramli MAM, Al-Turki YA. On the estimation of the optimum tilt angle of PV panel in Saudi Arabia. Renew Sustain Energy Rev 2016;65:626-34. https:// doi.org/10.1016/j.rser.2016.07.032.

[3] H. Hottel, A. Whillier, Evaluation of flat-plate solar collector performance, Trans. Conf. Use of Solar Energy 3 (Thermal Processes) Part 2.

[4] Klucher TM. Evaluation of models to predict insolation on tilted surfaces. Sol Energy 1979;23:111-4. https://doi.org/10.1016/0038-092X(79)90110-5.

[5] Hay JE, Davies JA. Calculations of the solar radiation incident on an inclined surface. Proceedings of first Canadian solar radiation data workshop. 1980. p. 59-72.

[6] Reindl DT, Beckman WA, Duffie JA. Diffuse fraction correlations. Sol Energy 1990;45(1):1-7. https://doi.org/10.1016/0038-092X(90)90060-P.

[7] Polo J, Fernández-Peruchena C, Gastón M. Analysis on the long-term relationship 
between DNI and CSP yield production for different technologies. Sol Energy 2017;155:1121-9. https://doi.org/10.1016/j.solener.2017.07.059.

[8] Bailek N, Bouchouicha K, Al-Mostafa Z, El-Shimy M, Aoun N, Slimani A, Al-Shehri S. A new empirical model for forecasting the diffuse solar radiation over Sahara in the Algerian Big South. Renew Energy 2018;117:530-7. https://doi.org/10.1016/j. renene.2017.10.081.

[9] Liu BY, Jordan RC. The interrelationship and characteristic distribution of direct, diffuse and total solar radiation. Sol Energy 1960;4(3):1-19. https://doi.org/10. 1016/0038-092X(60)90062-1.

[10] Page JK. The estimation of monthly mean values of daily total short wave radiation on vertical and inclined surfaces from sunshine records for latitudes $40 \mathrm{~N}-40 \mathrm{~S}$. Proc. UN conf. New sources energy, vol. 4. 1964. p. 378-87.

[11] Tuller SE. The relationship between diffuse, total and extra terrestrial solar radiation. Sol Energy 1976;18:259-63. https://doi.org/10.1016/0038-092X(76) 90025-6.

[12] Klein SA. Calculation of monthly average insolation on tilted surfaces. Sol Energy 1977;19:64-74. https://doi.org/10.1016/0038-092X(77)90001-9.

[13] Khorasanizadeh H, Mohammadi K, Goudarzi N. Prediction of horizontal diffuse solar radiation using clearness index based empirical models; A case study. Int $\mathrm{J}$ Hydrogen Energy 2016;41(47):21888-98. https://doi.org/10.1016/j.ijhydene. 2016.09.198.

[14] Gueymard CA, Ruiz-Arias JA. Extensive worldwide validation and climate sensitivity analysis of direct irradiance predictions from 1-min global irradiance. Sol Energy 2016;128:1-30. https://doi.org/10.1016/j.solener.2015.10.010.

[15] Orgill JF, Hollands KGT. Correlation equation for hourly diffuse radiation on a horizontal surface. Sol Energy 1977;19(4):357-9. https://doi.org/10.1016/0038092X(77)90006-8.

[16] Muneer T, Munawwar S. Improved accuracy models for hourly diffuse solar radiation. J Sol Energy Eng 2006;128(1):104-17. https://doi.org/10.1115/1 2148972.

[17] Lanini F. Division of global radiation into direct radiation and diffuse radiation Master's thesis Switzerland: Faculty of Science, University of Bern; 2010

[18] Bruno R. A correction procedure for separating direct and diffuse insolation on a horizontal surface. Sol Energy 1978;20(1):97-100. https://doi.org/10.1016/0038092X(78)90148-2.

[19] Erbs DG, Klein SA, Duffie JA. Estimation of the diffuse radiation fraction for hourly, daily and monthly-average global radiation. Sol Energy 1982;28(4):293-302. https://doi.org/10.1016/0038-092X(82)90302-4.

[20] Hawlader MN. Diffuse, global and extra-terrestrial solar radiation for Singapore. Int J Ambient Energy 1984;5(1):31-8. https://doi.org/10.1080/01430750.1984. 9675406.

[21] Muneer T, Hawas MM, Sahili K. Correlation between hourly diffuse and global radiation for New Delhi. Energy Convers Manag 1984;24(4):265-7. https://doi. org/10.1016/0196-8904(84)90004-9.

[22] Gueymard C. Progress in direct irradiance modeling and validation. Solar 2010 conf., phoenix. 2010.

[23] Jamil B, Akhtar N. Comparative analysis of diffuse solar radiation models based on sky-clearness index and sunshine period for humid-subtropical climatic region of India: a case study. Renew Sustain Energy Rev 2017;78(April):329-55. https://doi. org/10.1016/j.rser.2017.04.073.

[24] Gueymard CA. A review of validation methodologies and statistical performance indicators for modeled solar radiation data: towards a better bankability of solar projects. Renew Sustain Energy Rev 2014;39:1024-34. https://doi.org/10.1016/j rser.2014.07.117.

[25] Taylor KE. Summarizing multiple aspects of model performance in a single diagram. J Geophys Res 2001;106(D7):7183-92. https://doi.org/10.1029/2000JD900719.

[26] Boland J, Scott L, Luther M. Modelling the diffuse fraction of global solar radiation on a horizontal surface. Environmetrics 2001;12:1003-116. https://doi.org/10 1002/1099-095X(200103)12:2<103::AID-ENV447>3.0.CO;2-2.

[27] Ruiz-Arias JA, Alsamamra H, Tovar-Pescador J, Pozo-Vázquez D. Proposal of a regressive model for the hourly diffuse solar radiation under all sky conditions. Energy Convers Manag 2010;51(5):881-93. https://doi.org/10.1016/j.enconman. 2009.11.024.

[28] Spencer JW. A comparison of methods for estimating hourly diffuse solar radiation from global solar radiation. Sol Energy 1982;29(1):19-32. https://doi.org/10. 1016/0038-092X(82)90277-8.

[29] Ineichen P, Guisan O, Razafindraibe A. Indice de clarté, Mesures d' ensoleillement à Genève vol. 9. Switzerland: University of Geneva; 1984.

[30] Bakhsh H, Srinivasan R, Bahel V. Correlation between hourly diffuse and global radiation for Dhahran, Saudi Arabia. Sol Wind Technol 1985;2:59-61. https://doi. org/10.1016/0741-983X(85)90027-X.

[31] Hollands KGT. A derivation of the diffuse fraction's dependence on the clearness index. Sol Energy 1985;35(2):131-6. https://doi.org/10.1016/0038-092X(85) 90003-9.

[32] Al-Riahi M, Al-Hamdani N, Tahir K. An empirical method for estimation of hourly diffuse fraction of global radiation. Renew Energy 1992;2(4-5):451-6. https://doi. org/10.1016/0960-1481(92)90079-I.

[33] Bourges B. Climatic data handbook for Europe. Dordrecht: Kluwer; 1992.

[34] Chandrasekaran J, Kumar S. Hourly diffuse fraction correlation at a tropical location. Sol Energy 1994;53(6):505-10. https://doi.org/10.1016/0038-092X(94) 90130-T.

[35] Chendo MA, Maduekwe AAL. Hourly global and diffuse radiation of Lagos. NigeriaCorrel Some Atmos Parameters 1994;52(3):247-51. https://doi.org/10.1016/0038092X(94)90491-X.

[36] Maduekwe AA, Chendo MA. Atmospheric turbidity and the diffuse irradiance in Lagos, Nigeria. Sol Energy 1998;61(4):241-9. https://doi.org/10.1016/S0038-
092X(97)00020-0.

[37] Lam JC, Li DH. Correlation between global solar radiation and its direct and diffuse components. Build Environ 1996;31(6):527-35. https://doi.org/10.1016/03601323(96)00026-1.

[38] Hijazin MI. The diffuse fraction of hourly solar radiation for Amman/Jordan. Renew Energy 1998;13(2):249-53. https://doi.org/10.1016/S0960-1481(97) 00082-7.

[39] González JA, Calbó J. Influence of the global radiation variability on the hourly diffuse fraction correlations. Sol Energy 1999;65(2):119-31. https://doi.org/10 1016/S0038-092X(98)00121-2.

[40] De Miguel A, Bilbao J, Aguiar R, Kambezidis H, Negro E. Diffuse solar irradiation model evaluation in the North Mediterranean Belt area. Sol Energy 2001;70(2):143-53. https://doi.org/10.1016/S0038-092X(00)00135-3.

[41] Li DH, Lam JC. Analysis of solar heat gain factors using sky clearness index and energy implications. Energy Convers Manag 2001;42(5):555-71. https://doi.org/ 10.1016/S0196-8904(00)00082-0.

[42] Oliveira AP, Escobedo JF, Machado AJ, Soares J. Correlation models of diffuse solar-radiation applied to the city of São Paulo, Brazil. Appl Energy 2002;71(1):59-73. https://doi.org/10.1016/S0306-2619(01)00040-X.

[43] Ulgen K, Hepbasli A. Prediction of solar radiation parameters through clearness index for izmir. Turkey 2002;24(8):773-85. https://doi.org/10.1080/ 00908310290086680.

[44] Karatasou S, Santamouri M, Geros V. Analysis of experimental data on diffuse solar radiation in Athens, Greece, for building applications. Int J Sustain Energy 2003;23(1-2):1-11. https://doi.org/10.1080/0142591031000148597.

[45] Tsubo M, Walker S. Relationships between photosynthetically active radiation and clearness index at Bloemfontein, South Africa. Theor Appl Climatol 2005;80(1):17-25. https://doi.org/10.1007/s00704-004-0080-5.

[46] Soares J, Oliveira AP, Božnar MZ, Mlakar P, Escobedo JF, Machado AJ. Modeling hourly diffuse solar-radiation in the city of São Paulo using a neural-network technique. Appl Energy 2004;79(2):201-14. https://doi.org/10.1016/j.apenergy. 2003.11.004.

[47] Mondol JD, Yohanis YG, Smyth M, Norton B. Long-term validated simulation of a building integrated photovoltaic system. Sol Energy 2005;78(2):163-76. https:// doi.org/10.1016/j.solener.2004.04.021.

[48] Jacovides CP, Tymvios FS, Assimakopoulos VD, Kaltsounides NA. Comparative study of various correlations in estimating hourly diffuse fraction of global solar radiation. Renew Energy 2006;31(15):2492-504. https://doi.org/10.1016/j. renene.2005.11.009.

[49] Elminir HK, Azzam YA, Younes FI. Prediction of hourly and daily diffuse fraction using neural network, as compared to linear regression models. Energy 2007;32(8):1513-23. https://doi.org/10.1016/j.energy.2006.10.010.

[50] Boland J, Ridley B, Brown B. Models of diffuse solar fraction. Renew Energy 2008;33:575-84. https://doi.org/10.1016/j.renene.2007.04.012.

[51] Boland J, Ridley B. Models of diffuse solar fraction. Modeling solar radiation at the Earth's surface: recent advances Berlin: Springer; 2008. p. 193-219. https://doi. org/10.1007/978-3-540-77455-6_8.

[52] C. Furlan, A. P. Oliveira, Hourly diffuse solar radiation in the presence of clouds and other environmental parameters: the city of São Paulo, University of Padua, Italy.

[53] Mondol JD, Yohanis YG, Norton B. Solar radiation modelling for the simulation of photovoltaic systems. Renew Energy 2008;33(5):1109-20. https://doi.org/10. 1016/j.renene.2007.06.005.

[54] Moreno S, Silva M. Comparison of methodologies to estimate direct normal irradiation from daily values of global horizontal irradiation. 15th SolarPACES Conference, Berlin, Germany. 2009. p. 16136. (hal-00919055).

[55] Pagola Í, Gastón M, Fernández-Peruchena CM, Torres JL, Silva M, Ramírez L. Comparison and fitting of several Global-to-beam irradiance models in Spain. 15th SolarPACES conference, Berlin. 2009. p. 11724.

[56] Posadillo R, López Luque R. Hourly distributions of the diffuse fraction of global solar irradiation in Córdoba (Spain). Energy Convers Manag 2009;50(2):223-31. https://doi.org/10.1016/j.enconman.2008.09.042.

[57] Janjai S, Phaprom P, Wattan R, Masiri I. Statistical models for estimating hourly diffuse solar radiation in different regions of Thailand. Proceedings of the international conference on energy and sustainable development: issues and strategies (ESD 2010) (esd) 2010. p. 1-6. https://doi.org/10.1109/ESD.2010.5598771.

[58] Torres JL, De Blas M, García A, de Francisco A. Comparative study of various models in estimating hourly diffuse solar irradiance. Renew Energy 2010;35(6):1325-32. https://doi.org/10.1016/j.renene.2009.11.025.

[59] Chikh M, Mahrane A, Haddadi M. Modeling the diffuse part of the global solar radiation in Algeria. Energy Procedia 2012;18:1068-75. https://doi.org/10.1016/j. egypro.2012.05.121.

[60] Sánchez G, Cancillo ML, Serrano A. Adapting the Spencer model for diffuse solar radiation in Badajoz. Opt Pura Apl 2012;45(1):5-9. https://doi.org/10.7149/OPA. 45.1.5.

[61] Lee K, Yoo H, Levermore GJ. Quality control and estimation hourly solar irradiation on inclined surfaces in South Korea. Renew Energy 2013;57:190-9. https://doi.org/ 10.1016/j.renene.2013.01.028.

[62] Yao W, Li Z, Lu Y, Jiang F, Li C. New models for separating hourly diffuse and direct components of global solar radiation. Lect Notes Electr Eng 2014;261:653-63. doi:10/cj9s.

[63] Tapakis R, Michaelides S, Charalambides AG. Computations of diffuse fraction of global irradiance: Part 2 - neural Networks. Sol Energy 2016;139:723-32. https:// doi.org/10.1016/j.solener.2015.12.042.

[64] Abreu EFM, Canhoto P, Costa MJ. Contribution to the diffuse radiation modeling in Évora, Portugal. Workshop on earth Sciences, Évora. 2016.

[65] Marques Filho EP, Oliveira AP, Vita WA, Mesquita FL, Codato G, Escobedo JF, 
Cassol M, França JRA. Global, diffuse and direct solar radiation at the surface in the city of Rio de Janeiro: observational characterization and empirical modeling. Renew Energy 2016;91:64-74. https://doi.org/10.1016/j.renene.2016.01.040.

[66] Paulescu E, Blaga R. Regression models for hourly diffuse solar radiation. Sol Energy 2016;125:111-24. https://doi.org/10.1016/j.solener.2015.11.044.

[67] Abal G, Aicardi D, Alonso Suárez R, Laguarda A. Performance of empirical models for diffuse fraction in Uruguay. Sol Energy 2017;141:166-81. https://doi.org/10. 1016/j.solener.2016.11.030.

[68] Al-Najjar HMT, Al-Khazzar AAA. Experimental prediction of hourly diffuse solar radiation with clearness index in Baghdad ( Iraq ). Int J Comput Appl 2017;158(7):20-8

[69] Ohmura A, Dutton EG, Forgan B, Fröhlich C, Gilgen H, Hegner H, Heimo A, KönigLanglo G, McArthur B, Müller G, Philipona R, Pinker R, Whitlock CH, Dehne K, Wild M. Baseline surface radiation network (BSRN/WCRP): new precision radiometry for climate Research. Bull Am Meteorol Soc 1998;79(10):2115-36. https://doi.org/10. 1175/1520-0477(1998)079<2115:BSRNBW > 2.0.CO;2.

[70] Churchill SW, Usagi R. A general expression for the correlation of rates of transfer and other phenomena. AIChE J 1972;18(6):1121-8. https://doi.org/10.1002/aic. 690180606

[71] Canhoto P, Reis AH. Heat transfer in the thermal entry region of singly-connected tubes with uniform wall temperature. Jornadas de Física por ocasião da Jubilação do Professor Rui Namorado Rosa978-989-95091-2-2; 2010. p. 231-40.

[72] Canhoto P, Reis AH. Optimization of forced convection heat sinks with pumping power requirements. Int J Heat Mass Transf 2011;54:1441-7. https://doi.org/10. 1016/j.ijheatmasstransfer.2010.11.050.

[73] Canhoto P, Reis AH. Optimization of fluid flow and internal geometric structure of volumes cooled by forced convection in an array of parallel tubes. Int $\mathrm{J}$ Heat Mass Transf 2011;54:1441-7. https://doi.org/10.1016/j.ijheatmasstransfer.2011.05. 016.
[74] Driemel A, Augustine J, Behrens K, Colle S, Cox C, Cuevas-Agulló E, Denn FM, Duprat T, Fukuda M, Grobe H, Haeffelin M, Hodges G, Hyett N, Ijima O, Kallis A, Knap W, Kustov V, Long CN, Longenecker D, Lupi A, Maturilli M, Mimouni M, Ntsangwane L, Ogihara H, Olano X, Olefs M, Omori M, Passamani L, Pereira EB, Schmithüsen H, Schumacher S, Sieger R, Tamlyn J, Vogt R, Vuilleumier L, Xia X, Ohmura A, König-Langlo G. Baseline surface radiation network (bsrn): structure and data description (1992-2017). Earth Syst Sci Data 2018;10:1491-501. https://doi. org/10.5194/essd-10-1491-2018.

[75] Guide to meteorological instruments and methods of observation, vol. WMO - No. 8. World Meteorological Organization (WMO); 2017.

[76] C. Long, Y. Shi, The QCRad value added product: surface radiation measurement quality control testing, including climatology configurable limitsdoi:10.2172/ 1019540.

[77] Gueymard CA. Revised composite extraterrestrial spectrum based on recent solar irradiance observations. Sol Energy 2018;169:434-40. https://doi.org/10.1016/j. solener.2018.04.067.

[78] Reda I, Andreas A. Solar position algorithm for solar radiation applications. Sol Energy 2004;76(5):577-89. https://doi.org/10.1016/j.solener.2003.12.003.

[79] Behar O, Khellaf A, Mohammedi K. Comparison of solar radiation modelsand their validation under algerian climate - the case of direct irradiance. Energy Convers Manag 2012;98:236-51. https://doi.org/10.1016/j.enconman.2015.03.067.

[80] Despotovic M, Nedic V, Despotovic D, Cvetanovic S. Review and statistical analysis of different global solar radiation sunshine models. Renew Sustain Energy Rev 2015;52:1869-80. https://doi.org/10.1016/j.rser.2015.08.035.

[81] Jamil B, Akhtar N. Comparison of empirical models to estimate monthly mean diffuse solar radiation from measured data: case study for humid-subtropical climatic region of India. Renew Sustain Energy Rev 2017;77(December 2016):1326-42. https://doi.org/10.1016/j.rser.2017.02.057. 